ARTICLE

DOI: $10.1038 / \mathrm{s} 41467-017-01992-5$

\title{
Stem cell senescence drives age-attenuated induction of pituitary tumours in mouse models of paediatric craniopharyngioma
}

Jose Mario Gonzalez-Meljem (10 1,2, Scott Haston (1) 1, Gabriela Carreno', John R. Apps ${ }^{1}$, Sara Pozzi ${ }^{1}$, Christina Stache ${ }^{1}$, Grace Kaushal ${ }^{1}$, Alex Virasami ${ }^{3}$, Leonidas Panousopoulos ${ }^{1}$, Seyedeh Neda Mousavy-Gharavy ${ }^{1}$, Ana Guerrero ${ }^{4}$, Mamunur Rashid ${ }^{5}$, Nital Jani ${ }^{6}$, Colin R. Goding ${ }^{7}$, Thomas S. Jacques (1) 1,3, David J. Adams ${ }^{5}$, Jesus Gil ${ }^{4}$, Cynthia L. Andoniadou (D) ${ }^{8,9}$ \& Juan Pedro Martinez-Barbera ${ }^{1}$

Senescent cells may promote tumour progression through the activation of a senescenceassociated secretory phenotype (SASP), whether these cells are capable of initiating tumourigenesis in vivo is not known. Expression of oncogenic $\beta$-catenin in Sox $2+$ young adult pituitary stem cells leads to formation of clusters of stem cells and induction of tumours resembling human adamantinomatous craniopharyngioma (ACP), derived from Sox2- cells in a paracrine manner. Here, we uncover the mechanisms underlying this paracrine tumourigenesis. We show that expression of oncogenic $\beta$-catenin in Hesx $1+$ embryonic precursors also results in stem cell clusters and paracrine tumours. We reveal that human and mouse clusters are analogous and share a common signature of senescence and SASP. Finally, we show that mice with reduced senescence and SASP responses exhibit decreased tumour-inducing potential. Together, we provide evidence that senescence and a stem cellassociated SASP drive cell transformation and tumour initiation in vivo in an age-dependent fashion.

\footnotetext{
${ }^{1}$ Developmental Biology and Cancer Programme, Birth Defects Research Centre, UCL Institute of Child Health, London WC1N 1EH, UK. ${ }^{2}$ Basic Research Department, Instituto Nacional de Geriatría, Anillo Periférico 2767, Magdalena Contreras, 10200 Mexico City Mexico. ${ }^{3}$ Department of Histopathology, Great Ormond Street Hospital for Children, London WC1N 3JH, UK. ${ }^{4}$ Cell Proliferation Group, MRC Clinical Sciences Centre, Imperial College London, Hammersmith Campus, Du Cane Road, London W12 ONN, UK. ${ }^{5}$ Experimental Cancer Genetics, Wellcome Trust Sanger Institute, Hinxton, Cambridge CB10 1SA, USA. ${ }^{6}$ GOSgene, Genetics and Genomic Medicine, UCL Institute of Child Health, London WC1N 1EH, UK. ${ }^{7}$ Ludwig Institute for Cancer Research, Oxford University, Old Road Campus, Headington, Oxford OX3 7DQ, UK. ${ }^{8}$ Centre for Craniofacial and Regenerative Biology, King's College London, Guy's Hospital, Floor 27 Tower Wing, London SE1 9RT, UK. ${ }^{9}$ Department of Internal Medicine III, Technische Universität Dresden, Fetscherstaße 74,01307 Dresden Germany. Correspondence and requests for materials should be addressed to J.P.M.-B. (email: j.martinez-barbera@ucl.ac.uk)
} 
C ellular senescence defines a state of stable and long-term loss of proliferative capacity, but with retention of normal metabolic activity and viability ${ }^{1}$. The activation of the senescence programme acts as a potent tumour suppression mechanism through the activation of the p53 pathway and expression of cell cycle inhibitors (e.g. p21 (CDKN1A) and p16 $(\mathrm{CDKN} 2 \mathrm{~A}))^{2,3}$. The mitogenic stimuli caused by the expression of several oncogenic proteins, including mutant $\beta$-catenin,
$\mathrm{BRAF}^{\mathrm{V} 600 \mathrm{E}}$ or $\mathrm{KRAS}^{\mathrm{G} 12 \mathrm{D}}$, trigger DNA replication stress leading to DNA damage, activation of a DNA damage response (DDR) and the induction of senescence (named oncogene-induced senescence, OIS $)^{4,5}$. As a result, senescent cells activate a molecular programme characterised by the expression and secretion of a multitude of growth factors, matrix proteases and proinflammatory proteins collectively referred to as the senescenceassociated secretory phenotype (SASP) ${ }^{6}$. The composition and a

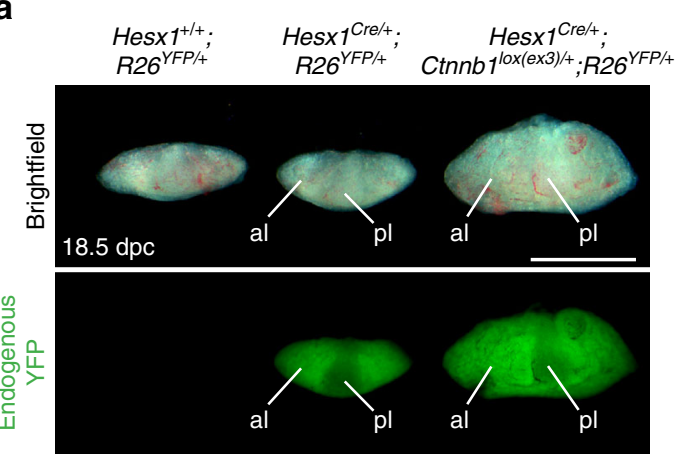

b

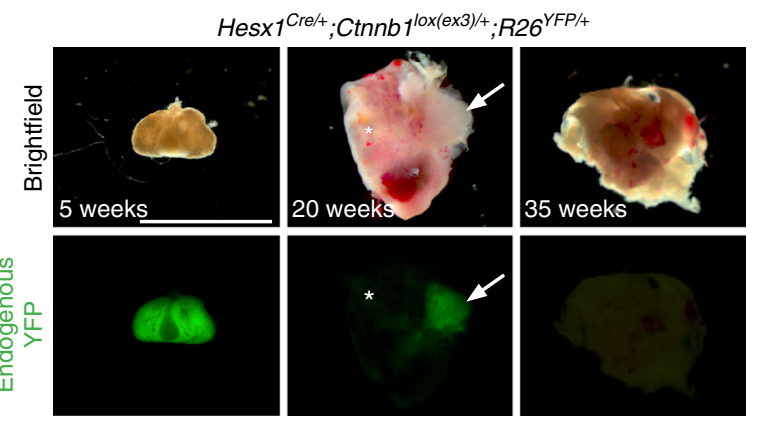

C

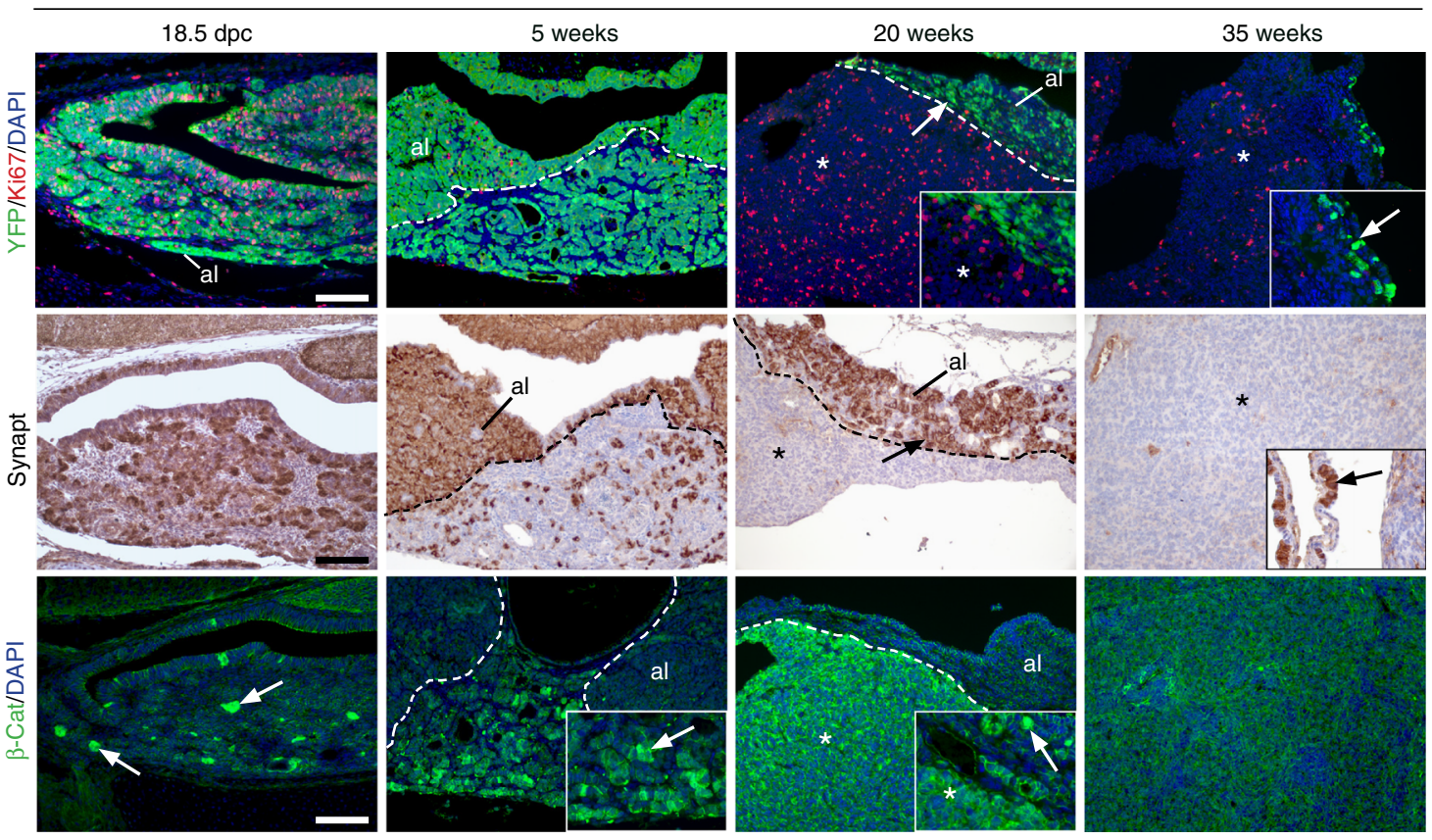

Fig. 1 Tumours in the $\mathrm{Hesx}_{7} \mathrm{Cre} /+^{+}$Ctnnb $7^{\mathrm{lox}(\mathrm{ex} 3) /+}, R 26^{\mathrm{YFP} /+}$ mice form non-cell autonomously after a latency period. a Brightfield and epifluorescence

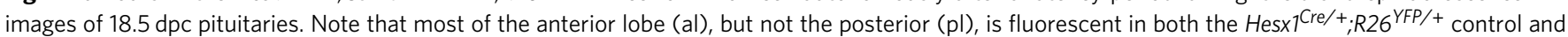
$\mathrm{Hes} \times 1^{\mathrm{Cre} /{ }_{+}} ; \mathrm{Ctnnb}^{\mathrm{lox}(\mathrm{ex} 3) /{ }_{+}} ; R 26^{\mathrm{YFP} /+}$ pituitaries. The mutant pre-tumoural pituitary is enlarged relative to controls. Scale bar: $1 \mathrm{~mm} . \mathbf{b}$ Brightfield and

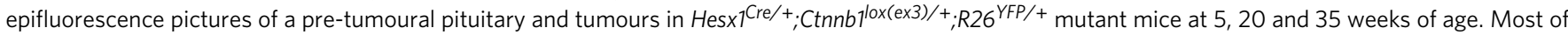
the tumour cells are not fluorescent and the YFP+ population (white arrow) is displaced to the periphery of the growing tumour mass (white asterisks). Scale bar: $5 \mathrm{~mm}$. c Histological characterisation of pre-tumoural pituitaries and developing tumours from $18.5 \mathrm{dpc}$ to $35 \mathrm{weeks}$ postnatally. At $18.5 \mathrm{dpc}$, most of the embryonic pituitary is comprised of YFP+ cells (revealed with anti-GFP immunostaining), but there are islands of YFP- cells (DAPI+ areas). Abundant Ki67+ cells are distributed throughout the pituitary at this stage. At 5 weeks of age, the pre-tumoural pituitary remains mostly quiescent with very few Ki67+ cells. Note the presence of a dorsal region resembling the normal pituitary tissue of the anterior lobe (al) and a ventral part where YFP- and YFP+ cells are intermingled. The boundary between dorsal and ventral regions is indicated with a dashed line. At 20 weeks, the YFP- population contains abundant Ki67+ cells and has expanded dramatically (white asterisks) displacing the mostly quiescent YFP+ cells to the periphery. Around 35 weeks, mutant mice reach a humane end-point and tumours are mostly YFP- (asterisk). Note that some YFP+ cells are observed in the periphery (white arrow). Immunohistochemistry against synaptophysin shows that whilst the anterior lobe cells (al) are positive, tumours do not express this endocrine marker (black asterisk). Immunostaining against $\beta$-catenin showing the presence of cell groups (clusters, white arrows) with nucleo-cytoplasmic accumulation of $\beta$-catenin mostly at $18.5 \mathrm{dpc}$ and 5 weeks of age. Subsequently, most of the tumour cells accumulate $\beta$-catenin (white asterisk). Results are representative of experiments conducted in at least three different mice for each stage. Scale bars: $100 \mu \mathrm{m}$ 
Table 1 Recurrently mutated genes in mouse and human ACP

Human ACP

\begin{tabular}{llll}
\hline Gene & cDNA change & Protein change & Variant classification \\
\hline ASCC2 & c. $1861 \mathrm{G}>\mathrm{A}$ & p.D621N & Missense \\
DNAJC3 & c. $1243 \mathrm{C}>\mathrm{T}$ & p.R415* & Nonsense \\
KIF1C & c. $2087 \mathrm{G}>\mathrm{A}$ & p.R696Q & Missense \\
PKD1L1 & c. 3862 C $>$ T & p.R1288C & Missense \\
TGFBI & c. $1286 \mathrm{C}>\mathrm{T}$ & p.A429V & Missense
\end{tabular}

${ }^{\star}$ Nonsense mutation

intensity of the SASP response can be affected by factors such as the senescence-inducing mechanism, cell type and time passed since senescence initiation, suggesting that the SASP is not a singular state $\mathrm{f}^{7-10}$. The activation of the SASP requires a persistent DDR and is mediated by the NF- $\kappa B$ and C/EBP $\beta$ pathways ${ }^{11}$. SASP-associated cytokines, IL-6 and IL-8, reinforce the senescence growth arrest, at least in some senescent cells ${ }^{12}, 13$, which is beneficial in cancer suppression. However, the paracrine activities of senescent cells through SASP activation can also promote tumourigenesis. Prominent or persistent SASP activation has been shown to: (1) disrupt cell-cell adhesion and induce epithelial-tomesenchymal transition and invasiveness ${ }^{14}, 15$; (2) cause local inflammation ${ }^{12,16}$; (3) modify tissue architecture ${ }^{17}, 18$; (4) facilitate development of hepatic cancer after carcinogen exposure ${ }^{19}$, ${ }^{20}$; (5) stimulate proliferation of nearby pre- and malignant cells both in vitro ${ }^{21}$ and in vivo when co-injected with senescent cells in xenograft mouse models ${ }^{17,18,22}$ and (6) favour the emergence of tumour-initiating cells in cell culture models ${ }^{23-26}$. This bulk of evidence demonstrates a pro-tumourigenic role for the SASP, but whether the SASP can induce cell transformation and tumour initiation of non-tumorigenic cells in vivo remain less clear.

We have previously shown that the expression of a degradationresistant form of $\beta$-catenin in Rathke's pouch, the embryonic primordium of the anterior pituitary gland $\left(\mathrm{Hes} \times 1^{\mathrm{Cre} /+}\right.$; Ctnnb1 $1^{\text {lox }(e x 3) /+}$ mice $)^{27}$, or in Sox $2+$ adult pituitary stem cells $\left(\text { Sox2 } 2^{\text {CreERT2/+}} \text {; Ctnnb1 } 1^{\text {lox }(e x 3) /+} \text { mice }\right)^{28}$ leads to the formation of tumours that resemble human adamantinomatous craniopharyngioma (ACP). Interestingly, targeting expression of this mutant $\beta$-catenin to cell-lineage progenitors or differentiated cells in the developing pituitary is not tumourigenic, suggesting that the oncogenic effect requires an undifferentiated stem/cell precursor $^{27}$. ACPs are clinically aggressive tumours associated with high morbidity and significant premature mortality ${ }^{29}$. Most human ACPs carry mutations in $\beta$-catenin leading to the overactivation of the $\mathrm{WNT} / \beta$-catenin pathway ${ }^{30-33}$. In agreement with this finding, cells showing nucleo-cytoplasmic accumulation of $\beta$ catenin and activation of the WNT pathway are present in mouse and human tumours, commonly grouped in whorl-like structures, named 'cell clusters', near the invasive front ${ }^{29}$. These cell clusters are not found in any other type of pituitary tumours ${ }^{34}$, express stem cell markers ${ }^{27,35}$ and have been proposed to play a critical role in controlling the infiltrative behaviour of surrounding tumour cells ${ }^{36}$.

Although murine clusters derive from mutant Sox $2+$; S100B+ adult pituitary stem cells expressing oncogenic $\beta$-catenin ${ }^{28}$, this population is not the cell-of-origin of the tumours, suggesting a non-cell autonomous role during tumourigenesis. Currently, the molecular and cellular mechanisms underlying the protumorigenic role of this peculiar cell population remain to be discovered. In this study, we demonstrate through molecular and genetics approaches that murine and human clusters are functionally equivalent structures, which show a molecular signature of cellular senescence and a SASP. Our results indicate that tumour induction only occurs in the presence of robust SASP activation, therefore providing evidence for a role of senescence and SASP in tumour initiation in vivo.

\section{Results}

Pituitary embryonic precursors can induce paracrine tumours. We previously showed that the expression of oncogenic $\beta$-catenin in Sox $2+$ pituitary stem cells in Sox $2^{\text {CreERT2/+}} ;$ Ctnnb $11^{\text {lox }(e \times 3) /+}$; $R 26^{Y F P /+}$ mice at 4-6 weeks of age results in the formation of pituitary tumours in a non-cell autonomous manner ${ }^{28}$. We sought to investigate whether a similar mechanism may underlie the development of pituitary tumours in $\mathrm{Hes}_{1} 1^{\mathrm{Cre} /+} ; \mathrm{Ctnnb1} 1^{\text {lox }}$ ${ }^{(e x 3) /+} ; R 26^{Y F P /+}$ mice, another model for human ACP, in which oncogenic $\beta$-catenin is expressed in the embryonic pituitary precursors that normally give rise to all the cell lineages of the mature pituitary gland, including Sox $2+$ stem cells ${ }^{27}$.

The pituitary gland of the Hes $x 1^{\mathrm{Cre} /+} ; \mathrm{Ctnnb} 1^{\text {lox(ex3)/+}} ; \mathrm{R}_{2} 6^{\mathrm{YFP} /+}$ mice at $18.5 \mathrm{dpc}$ was enlarged in comparison with the $\mathrm{Hes} \times \mathrm{I}^{\mathrm{Cre} /+}$; $R 26^{Y F P /+}$ controls, but did not develop tumours at this stage and will be hereafter referred to as a 'pre-tumoural' pituitary (Fig. 1a). The vast majority of the cells in the developing anterior pituitary were YFP+ with abundant Ki67 expression, as expected at this developmental stage (Fig. 1c). After a variable latency period of several weeks (median age of 17.7 weeks, range $8.3-35.3$ weeks ${ }^{37}$ ), the YFP+ population was progressively replaced by actively proliferating YFP-;Ki67+ cells (Fig. 1b, c). Eventually, in fully developed tumours (i.e. when mice reached the humane endpoint), none or only scarce YFP+ cells could be observed in the periphery of the majority of the tumours ( 8 out of 11 ), which were comprised mostly of YFP- cells. Notably, Ki67 expression was mostly observed in YFP- cells at this point (Fig. 1c). Similar results were obtained from $\mathrm{Hes}_{1} 1^{\mathrm{Cre} /+} ; \mathrm{Ctnn} b 1^{\text {lox }(e \times 3) /+}$; $R 26^{m T m G /+}$ mice, in which 4 out of 5 tumours contained large tdTomato+ areas, indicating that tumour cells were not recombined (Supplementary Fig. 1). $\beta$-catenin-accumulating cell clusters were evident at pre-tumoural stages and as expected were YFP+ (Fig. 1c) and Ki67-27. As tumours developed, $\beta$-catenin expression was elevated in YFP- cells and clusters were no longer identifiable (e.g. after 20 weeks) (Fig. 1c). Additionally, YFP- cells were synaptophysin-, suggesting that these are not neuroendocrine cells (Fig. 1c).

Exome sequencing of 15 murine tumours revealed the presence of a total of 272 mutations (average 18.13 mutations per tumour; Supplementary Data 2) of which 131 were in coding regions (average 8.73 mutations per tumour; Supplementary Data 2). Of a selection of 14 of these 131 mutations, we validated 12 by Sanger sequencing (i.e. they were identified in the same DNA used for exome sequencing) (Supplementary Data 2). Only three genes were recurrently mutated in either two or three separate tumours and no recurrent mutations were found present in all 15 tumours (Supplementary Data 2). A low mutational burden with few recurrent mutated genes, other than CTNNB1, has been described 
a

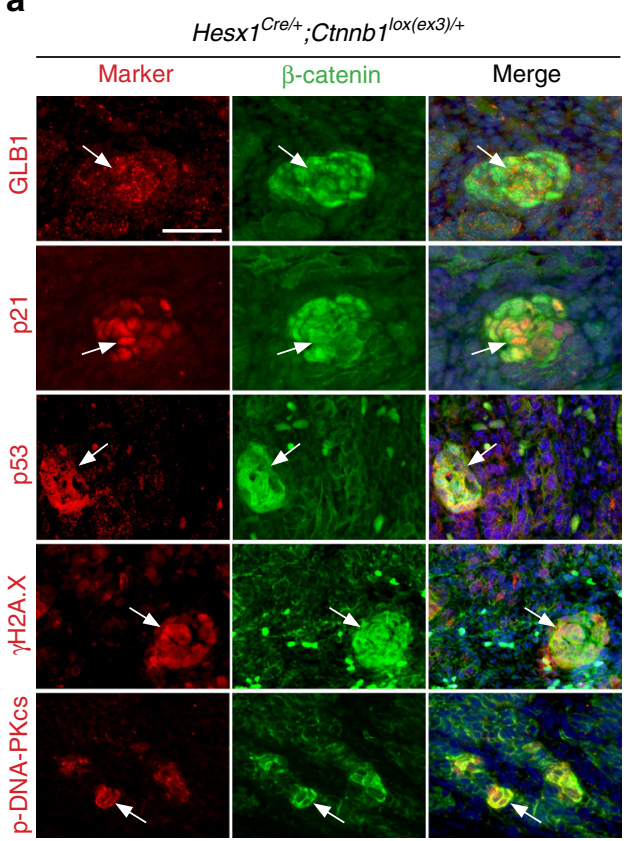

b

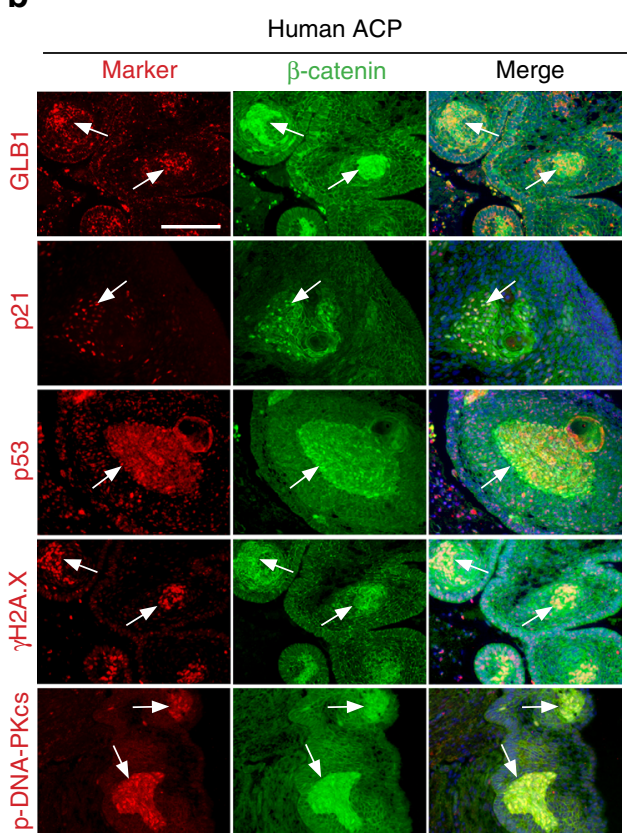

Fig. $2 \beta$-catenin-accumulating cluster cells in mouse and human ACP express senescence markers. Double immunostaining on histological sections of Hesx $1^{C r e /+} ;$ Ctnnb1ox(ex3)/+ pre-tumoural pituitaries at $18.5 \mathrm{dpc}$ (a) and human ACP tumours (b). Note that cluster cells (arrows) express lysosomal enzymes such as GLB1, encoding senescence-associated $\beta$-galactosidase, the cell cycle regulators p21 and p53, as well as the DNA damage and DNA damage response markers gamma histone $2 \mathrm{AX}$ and phospho-DNA-PKcs, respectively. Results are representative from experiments conducted in six to eight mice and 23 human ACP tumours. Normal brain was used as negative control. Scale bars: a $50 \mu \mathrm{m} ; \mathbf{b} 100 \mu \mathrm{m}$

in human $\mathrm{ACP}^{33}$. To determine if these changes are representative of the mutagenic process occurring in human ACP, we carried out comparisons with the published exome sequence results of 12 human $\mathrm{ACPs}^{33}$. This revealed 12 genes carrying mutations in both human and mouse tumours, but none of these mutations were identical between species (Supplementary Data 2). Five of these 12 mutated genes harboured non-silent/intronic mutations in both species (Table 1). The functional significance of these mutations is currently unknown.

Together, these findings demonstrate that the activation of oncogenic $\beta$-catenin in Hesx $1+$ pituitary embryonic precursors leads to cell transformation and formation of somatic mutationsbearing tumours, which are mostly not derived from the Hes $x 1$ cell lineage. Additionally, our time course analysis reveals that tumour initiation requires a latency period of several weeks and is preceded by formation of $\beta$-catenin-accumulating cell clusters in the pre-tumoural pituitary.

ACP clusters contain senescent cells with an activated SASP. The observation that cluster cells do not divide led us to hypothesise that the expression of mutant $\beta$-catenin may result in oncogeneinduced senescence. We analysed the expression of a panel of senescence-associated markers by immunostaining in mutant mouse embryos at $18.5 \mathrm{dpc}$ and 23 different human ACP samples to assess whether hallmark senescence features were present in cluster cells including: (i) cell cycle arrest; (ii) DNA damage and activation of a DDR and (iii) increased lysosomal content ${ }^{1,38}$.

Cluster cells were negative for the mitotic marker phosphohistone $\mathrm{H} 3$ and did not incorporate 5-ethynyl-2'-deoxyuridine $(\mathrm{EdU})$, a specific indicator of $\mathrm{S}$ phase. Cell death was not detected by caspase 3 immunostaining (Supplementary Fig. 2). Human $\beta$ catenin-accumulating cluster cells are also negative for Ki67 and caspase $3^{36,39}$. Murine and human clusters exhibited marked expression of p53 (TP53), p21 (CDKN1A) and p16 (CDKN2A), all crucial effectors of cell cycle arrest ${ }^{38}$ (Fig. 2a, b; Supplementary
Fig. 3a, b). Cluster cells in mouse and human ACP expressed $\gamma$ $\mathrm{H} 2 \mathrm{AX}$, suggesting the presence of DNA damage ${ }^{40}$; as well as phospho-DNA-PKcs, PARP1 and phospho-ATM, therefore revealing DDR activation ${ }^{41,42}$ (Fig. 2a, b; Supplementary Fig. 3c, d). The senescence-associated $\beta$-galactosidase (SA- $\beta$ gal) staining is a classical assay to identify senescent cells by detecting the presence of the lysosomal enzyme $\beta$-D-galactosidase ${ }^{43}$, the product of the GLB1 gene ${ }^{44}$. SA- $\beta$ gal staining on Hes $\times 1^{\mathrm{Cre} /+}$; Ctnnb1 $1^{l o x(e \times 3) /+}$ mutant pituitaries (Supplementary Fig. 3e) and double immunofluorescence for $\beta$-catenin and GLB1 revealed the expression of GLB1 in the cell clusters in both mouse and human ACP (Fig. 2a, b). LAMP1, LAMP2 and lysozyme C expression were also upregulated in cluster cells, suggesting the enlargement of the lysosomal compartment, another feature of senescent cells (Supplementary Fig. 3f, g) ${ }^{44,45}$.

The activation of the NF- $\kappa B$ pathway is required for survival of senescent cells and activation of $\mathrm{SASP}^{11}$. As a read out of an activated NF- $\kappa B$ pathway, we assessed the presence of phospho$\mathrm{I} \kappa \mathrm{B} \alpha$, as phosphorylation at Ser32 and Ser36 of I $\mathrm{B} \mathrm{B} \alpha$ is followed by proteasome-mediated degradation and pathway activation ${ }^{46}$. Mouse and human clusters expressed high levels of $\mathrm{p}-\mathrm{I} \kappa \mathrm{B} \alpha$ (Fig. 3a, b). RELA/p65 and NEMO, two crucial components of the NF- $\mathrm{BB}$ pathway, were broadly expressed throughout the tissue, including cluster cells of both species (Fig. 3a, b). Finally, to determine if senescence and the SASP response are initiated together with cluster formation and prior to any tumour initiation, we turned to the early stages of mutant pituitary development in the embryonic mouse model. Analysis of Hes $x 1^{\mathrm{Cre} /+}$;Ctnnb1 ${ }^{\operatorname{lox}(\operatorname{ex} 3) /+}$ embryonic pituitaries from 10.5 to $18.5 \mathrm{dpc}$ revealed a dynamic response for the different aspects of senescence in the cluster cells. Proliferation in $\beta$-cateninaccumulating cluster cells, as assessed by Ki67 and EdU incorporation, was higher at $10.5 \mathrm{dpc}$ than $18.5 \mathrm{dpc}$, whilst expression of the cell cycle inhibitors p16 and p21 was detected in the majority of the cluster cells from $10.5 \mathrm{dpc}$ (Supplementary 
a

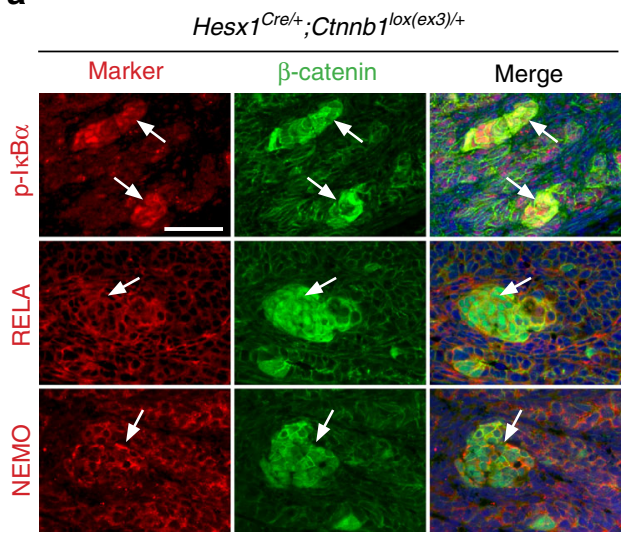

b

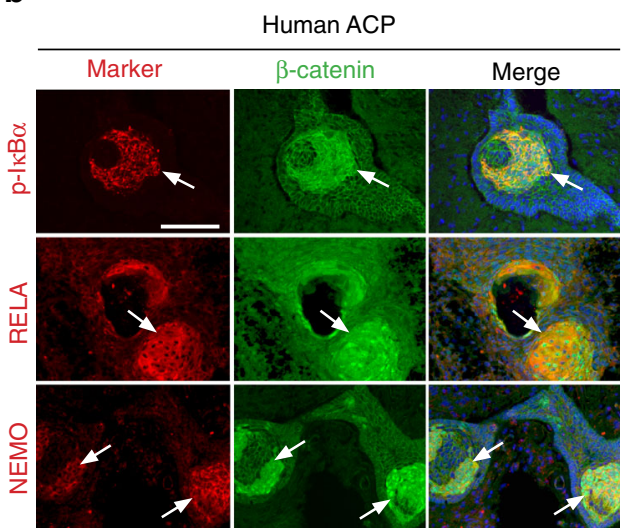

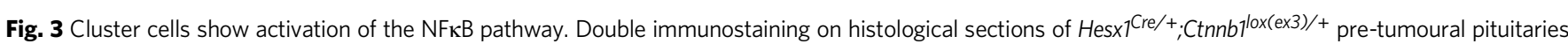
at $18.5 \mathrm{dpc}(\mathbf{a})$ and human $\mathrm{ACP}$ tumours (b). Phospho- $\mid \mathrm{kB} \alpha$, a readout of activated NFKB pathway, as well as RELA and NEMO, two components of this pathway, are expressed in cluster cells (white arrows). Results are representative of experiments conducted in four to six mice. Scale bar: $\mathbf{a} 50 \mu \mathrm{m} ; \mathbf{b} 100 \mu \mathrm{m}$

Fig. $4 \mathrm{a}, \mathrm{b})$. The DDR and NF- $\mathrm{BB}$ pathways became active by 10.5-12.5 dpc (Supplementary Fig. 4c), whereas expansion of the lysosomal compartment occurred later in development around 16.5-18.5 dpc (Supplementary Fig. 4a, b, d). Therefore, the senescence/SASP response is initiated at early stages of development, soon after the emergence of tumours.

SASP activation results in alterations in normal tissue architecture and increased angiogenesis ${ }^{6}$, therefore, we sought to analyse the tumour microenvironment in $\mathrm{Hes} \times 1^{\mathrm{Cre} /+}$; Ctnnb1 $1^{l o x(e x 3) /+}$ pituitaries at $18.5 \mathrm{dpc}$. In controls, immunostaining against endomucin (EMCN), a sialoglycoprotein routinely used as a marker of endothelial cells ${ }^{47}$, revealed the presence of cells with a thin and elongated morphology surrounding the lumen of blood vessels in the anterior lobe of the control pituitaries (Fig. 4a). In contrast, Hes $x 1^{\mathrm{Cre} /+} ; \mathrm{Ctnn}_{\mathrm{b}} 1^{\mathrm{lox}(\mathrm{ex} 3) /+}$ pituitaries displayed a drastic expansion of the EMCN+ population, which formed large and dense groups of cells without the presence of a lumen (Fig. 4a). Double immunostaining against YFP and EMCN in the Hes $\times 1^{C r e /+} ; C_{n n n b 1}$ lox(ex3)/+$; R_{2} 26^{Y F P /+}$ pituitaries revealed mutually exclusive staining with the EMCN cells occupying almost entirely the YFP- areas (Fig. 4b). Expression of extra-cellular matrix proteins such as fibronectin and laminin was altered in the Hes $x 1^{C r e /+} ; C_{n n n b 1}{ }^{l o x}($ ex3 $) /+$ pre-tumoural pituitaries when compared with controls (Fig. 4a). Interestingly, EMCN+ cells frequently formed ring-like structures surrounding $\mathrm{YFP}+$ cells, which resembled the $\beta$-cateninaccumulating clusters. Triple immunostaining against SOX9, $\beta$-catenin and EMCN confirmed that the cells directly surrounding the clusters are SOX9+;EMCN+ (Fig. 4c). Together, these results suggest that cluster cells activate a senescence and SASP programme leading to changes in the cellular microenvironment of the pre-tumoural pituitary.

ACP clusters show a senescence/SASP molecular signature. The expression of oncogenic $\beta$-catenin in Sox $2+$ pituitary stem cells in Sox $2^{\mathrm{CreERT}^{2 /+}} ; \mathrm{Ctnnb}^{\text {lox }(\mathrm{ex} 3) /+} ; \mathrm{R} 6^{\mathrm{YFP} /+}$ mice at $4-6$ weeks of age results in cluster formation and induction of paracrine tumours ${ }^{28}$. However, the molecular signature of the cluster cells in Sox $2^{\text {CreERT2/+}}$;Ctnnb1 $1^{\text {lox }(e \times 3) /+} ; R 26^{Y F P /+}$ mice has not yet been revealed. To this end, we first induced Sox2 $2^{C r e E R T 2 /+}$; Ctnnbl ${ }^{\text {lox }(e x 3) /+} ; R 26^{Y F P /+}$ mice at 4 weeks of age by tamoxifen administration and analysed the pituitaries 4 weeks later. Following dissociation into single-cell suspensions, YFP+ and YFP- cells were isolated by flow sorting and subjected to qRT-PCR analysis. This revealed a gene expression profile consistent with the presence of $\beta$-catenin-accumulating cluster cells in the YFP+ population (Supplementary Fig. 5b).

Next we sought to reveal the molecular signature of cluster cells relative to normal Sox $2+$ cells not expressing oncogenic $\beta$-catenin. Sox $2^{\mathrm{CreERT} 2 /+} ;$ Ctnnb1 $1^{\text {lox }(e x 3) /+} ; \mathrm{R}_{2} 6^{\mathrm{YFP} /+}$ mutant and Sox $2 \mathrm{CreERT2/+}$; $R 26^{Y F P /+}$ control mice were tamoxifen induced at 4 weeks of age and pituitaries dissociated and subjected to RNA sequencing 4 weeks later. The expression data for all of the 27,179 genes are included in Supplementary Data 3 and the demonstration of a successful experimental approach in Supplementary Fig. 5a. Of note, differential expression studies demonstrated the upregulation of several members of the hedgehog $(\mathrm{HH})$, fibroblast growth factor (FGF), bone morphogenetic protein (BMP), wingless-related integration site (WNT) and transforming growth factor $\beta$ (TGF $\beta$ ) families as well as CXC chemokines and interleukins (Supplementary Data 3). These results support the notion that cluster cells are signalling hubs in the pre-tumoural pituitary.

To assess in an unbiased manner whether cluster cells in Sox $2^{\mathrm{CreERT2} /+} ; \mathrm{Ctnnb}^{\mathrm{lox}(\mathrm{ex} 3) /+}$ and $\mathrm{Hes} \times 1^{\mathrm{Cre} /+} ; \mathrm{Ctnnb} 1^{\text {lox(ex3)/+ }}$ (previously characterised ${ }^{48}$ ) mice activate senescence and a SASP, we specifically compared the expression profiles of the murine clusters with established molecular signatures for OIS and SASP obtained from human IMR90 ER:RAS fibroblasts. These cells express a Steroid Receptor-KRAS ${ }^{\mathrm{G} 12 \mathrm{~V}}$ chimaeric fusion protein that triggers growth arrest, senescence and $\mathrm{SASP}^{13}$. Gene set enrichment analysis (GSEA) showed that the gene expression profiles of the clusters in both mouse models were significantly enriched for OIS and SASP genes (Fig. 5a, c). This was further validated by qRT-PCR (Fig. 5b, d). SASP activation was further investigated using semi-quantitative ELISA cytokine arrays, which demonstrated the upregulation of several SASP-related proteins, such as ICAM1, bFGF, IL1A, IFNG, OPN, IL6, IGFBL6, MMP3, MMP2, RNAKL1, CXCL1, CXCL11, IL7, TIMP2, VEGFR1 and CCL11) (Fig. 5e).

To determine whether these findings were relevant to human $\mathrm{ACP}$, we assessed the degree of molecular equivalence between mouse and human ACP clusters. We recently analysed the molecular signatures of specific tumour cell compartments in human ACP including the $\beta$-catenin-accumulating cell clusters, by combining laser-capture microdissection and RNASequencing (J.R. Apps and J.P. Martinez-Barbera, manuscript submitted, Array Express accession code: E-MTAB-5266). Hierarchical clustering analysis revealed the grouping of

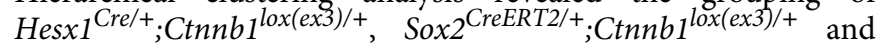
human clusters, suggesting similar molecular profiles (Fig. $5 \mathrm{~g}$ ), while GSEA confirmed that human clusters also have a molecular 
a

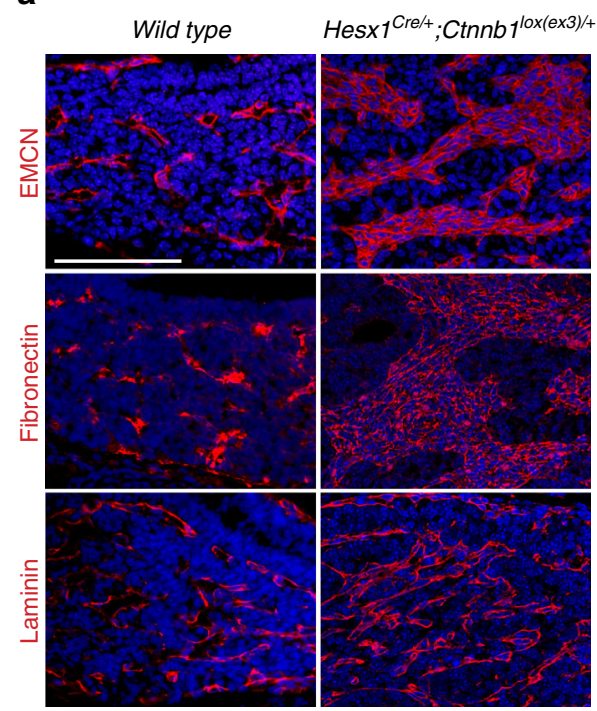

b

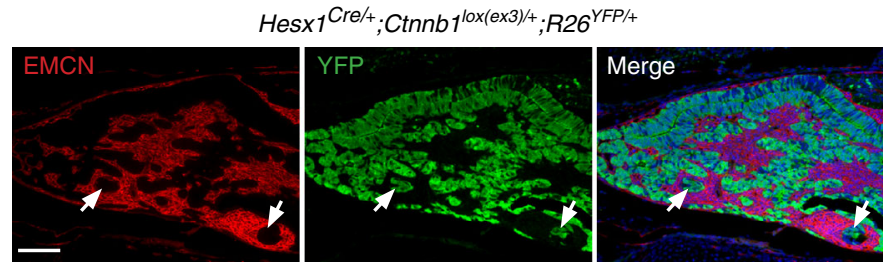

C

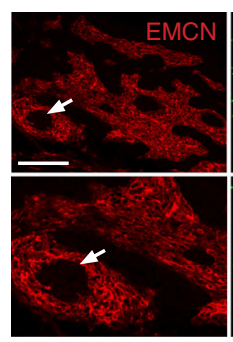

$\operatorname{Hes\times 1}{ }^{\mathrm{Cre} /+} ;$ Ctnnb $^{\text {Iox(ex3)/+ }}$

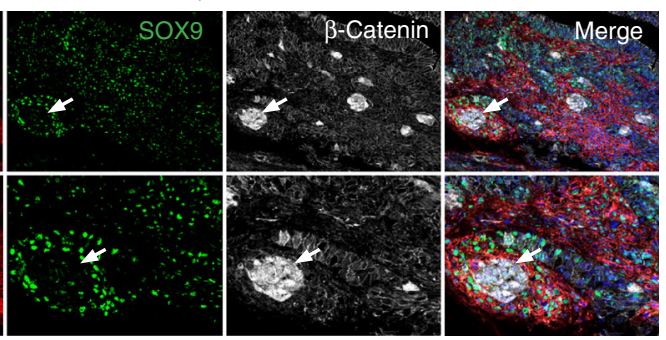

Fig. 4 Tissue microenvironment changes in the pre-tumoural pituitary of $\operatorname{Hesx}_{7}{ }^{\mathrm{Cre} /+}$; $\operatorname{Ctnnb} 7^{\mathrm{lox}(\mathrm{ex} 3) /+}$ mutants. a Immunostaining against endomucin $(E M C N)$, fibronectin and laminin revealing changes in the parenchyma of the pre-tumoural pituitary at $18.5 \mathrm{dpc}$. Scale bar: $100 \mu \mathrm{m}$. $\mathbf{b}$ Mutually exclusive staining of YFP+ (detected with an anti-GFP antibody) and EMCN+ cells. Note the presence of YFP+ cells (arrows) surrounding a population of EMCN+ cells. Scale bar: $100 \mu \mathrm{m}$. c Triple immunostaining showing the co-expression of EMCN and SOX9 in large group of cells around the $\beta$-catenin-accumulating clusters (arrows). Results are representative of experiments conducted in six mice. Scale bar: $100 \mu \mathrm{m}$

signature of senescence and SASP (Fig. 5f). Moreover, GSEA analysis of Hes $x 1^{\mathrm{Cre} /+}$; Ctnnb1 $1^{\text {lox(ex3)++ }}$ and Sox $2^{\mathrm{CreERT} 2 /+}$; Ctnnb1 $1^{l o x(e x 3) /+}$ cluster gene expression profiles showed a significant enrichment for the top 100 and 500 most upregulated genes in human clusters (Fig. 5h, i). Together, these analyses support that human and mouse clusters are equivalent structures, sharing a common signature of senescence and SASP.

Aged Sox2+ stem cells show reduced tumour-inducing potential. We sought to determine whether the age at which Sox $2^{\text {CreERT2/+}}$;Ctnnb $1^{\text {lox }(e x 3) /+}$ mice were targeted to express the oncogenic $\beta$-catenin may have an effect on paracrine tumourigenesis.

Sox $2^{\text {CreERT2/+}} ;$ Ctnnb1 $1^{\text {lox(ex3)/+ }}$ mutant mice were tamoxifen induced at either 1 month, when pituitary stem cells are still actively supporting organ growth, or at 6-9 months of age when contribution from Sox $2+$ stem cells to homeostasis is low. Pituitaries were analysed at 3 months post induction and assessed for tumour formation. Histological analysis revealed that $15 / 15$ mice that were induced at 1 month of age developed pituitary tumours with a total of 48 individual lesions (average 2-4 tumours per pituitary). However, only 3 out of 17 mice induced at 6-9 months of age developed pituitary tumours with only five individual lesions observed ( $p<0.0001$; Table 2).

We decided to explore whether the reduced tumour burden in older Sox $2^{\text {CreERT2/+}}$;Ctnnb1 ${ }^{\text {lox(ex3)/+ }}$ mice may be related to an attenuated senescence and SASP response in the $\beta$-cateninaccumulating clusters. To achieve this, $\mathrm{YFP}+$ cells were isolated by flow cytometry from Sox $2^{C r e E R T 2 /+} ; C \operatorname{Cnnb} 1^{l o x(e \times 3) /+} ; R_{26} 6^{Y F P /+}$ mutant and Sox $2^{\mathrm{CreERT2/+}} ; \mathrm{R}_{2} 6^{\mathrm{YFP} /+}$ control mice that were induced at either one month or 6-9 months of age (Fig. 6a). Relative to age-matched control YFP+ cells, YFP+ cells from older mice activated the WNT pathway and a senescence/SASP programme (Fig. 6d). Although both age groups contained Ki67-/p21+ $\beta$-catenin-accumulating clusters (Fig. 6b), the average number of cells in the clusters of young-induced Sox $2^{\text {CreERT2/+}}$;Ctnnb1 $1^{\text {lox(ex } 3) /+} ; R 26^{Y F P /+}$ mutant mice was 3.16 \pm 1.57 vs. $1.95 \pm 1.12$ in older-induced animals, affirming that clusters from older mice were smaller in size $(p<0.0001$, Student's $t$ test; 57 clusters counted in three young Sox 2 CreERT2/ ${ }^{+}$;Ctnnbl ${ }^{\text {lox }(e x 3) /+} ; R 26^{Y F P /+}$ pituitaries and 94 clusters counted in six older Sox $2^{C r e E R T 2 /+} ; C t n n b 1^{l o x(e x 3) /+} ; R 26^{Y F P /+}$ pituitaries). In addition, increased Ki67 index was observed only in non-cluster cells in the younger, but not in the older Sox $2^{\text {CreERT2/+ }}$ Ctnnb $1^{\text {lox }}$ ${ }^{(e \times 3) /+} ; R 26^{Y F P /+}$ pituitaries, suggesting that Sox $2+$ cluster cells from younger mice can induce a proliferative response in surrounding cells (Fig. 6c).

Importantly, comparative analysis between the YFP+ fractions of Sox $2^{\text {CreERT2/+}}$;Ctnnb1 $1^{\text {lox(ex3)/+}} ; R 26^{Y F P /+}$ mutants revealed a significant reduction in the expression of senescence and SASP factors in mice induced at 6-9 months relative to the one monthinduced mice (Fig. 6e). We also observed an age-dependent increase in the expression of senescence-associated markers, such as p16 (Cdkn2a) and p53 (Trp53), in non-mutant Sox2+ cells in Sox $2^{C r e E R T 2 /+} ; R_{2} 6^{Y F P /+}$ control pituitaries, which may underlie the ameliorated senescence/SASP response of older relative to younger mutant Sox $2+$ stem cells (Supplementary Fig. 6). Together, these analyses demonstrate that expression of oncogenic $\beta$-catenin in Sox $2+$ stem cells in 6-9 months old mice is less tumourigenic and leads to weakened senescence and SASP responses with formation of smaller $\beta$-catenin-accumulating clusters.

Apc-deficient clusters lack tumour-inducing potential. The data previously described in $\mathrm{Hes} \mathrm{1}^{\mathrm{Cre} /+} ; \mathrm{Ctnn}$ b $1^{\text {lox(ex3)/ }+}$ and Sox $2^{C r e E R T 2 /+} ;$ Ctnnb $1^{\text {lox(ex3)/+ }}$ mice are compatible with a model whereby senescent cells in the $\beta$-catenin-accumulating clusters of the pre-tumoural pituitary may initiate tumourigenesis by activating a robust SASP programme. Therefore, we sought to explore whether the reduction or ablation of senescent cells may prevent or delay tumourigenesis.

The senolytic compounds ABT-263 and ABT-737 have been shown to selectively ablate senescent cells in vitro and in vivo 49,50 . In agreement with these findings, ex vivo culture of $18.5 \mathrm{dpc} \mathrm{Hes} \times 1^{\mathrm{Cre} /+} ;$ Ctnnb $1^{\text {lox(ex3)/+ }}$ pre-tumoural pituitaries in the presence of ABT-263 and ABT-737 revealed a reduction in 
the average size of the clusters, but this did not reach significance (number of cells per cluster: vehicle $2.46 \pm 1.26,41$ clusters counted from three pituitaries; ABT-263, $1.4 \pm 0.49, p=0.47,40$ clusters counted from four pituitaries; ABT-737, $1.8 \pm 0.62, p=$ $0.70,38$ clusters counted from three pituitaries; Student's $t$ test) (Supplementary Fig. 7c, d). In contrast, treatment of IMR90 ER: RAS senescent fibroblasts with these two senolytic agents significantly increased cell death relative to control non-

a

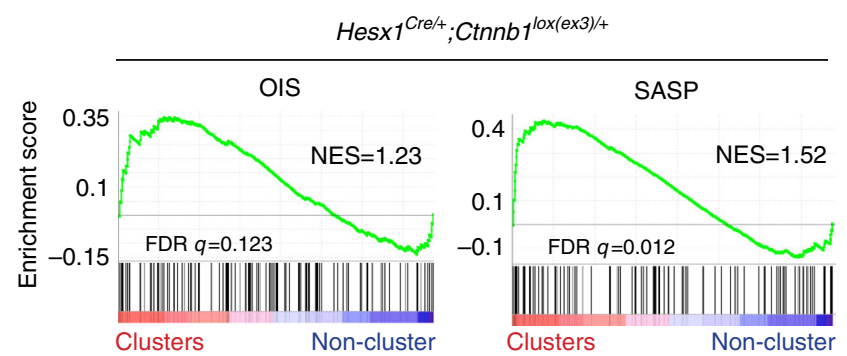

C

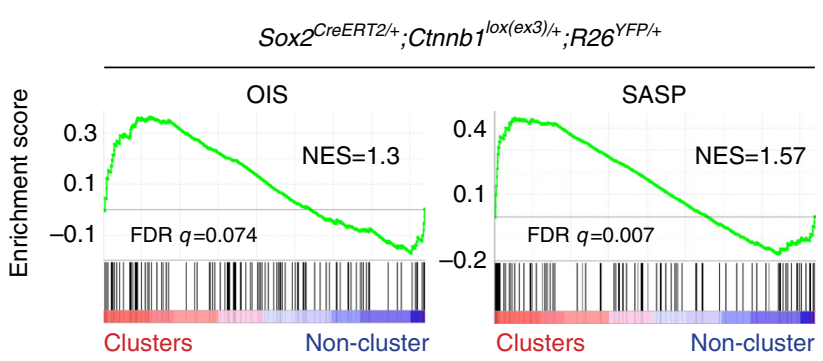

e

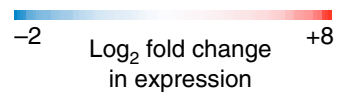

senescent cells (Supplementary Fig. 7a, b). The distinct target cell type (i.e. epithelial cluster cell rather than fibroblast) may confer a resistance to these drugs and, additionally, drug delivery and dose may require further optimisation. The drugs target genes $B c l 2, B c l-x l$ and $B c l-w$ are all expressed in the cluster cells, but not upregulated.

Since chemical ablation of senescent cells was not strong enough to reduce significantly the size of the clusters, we sought b

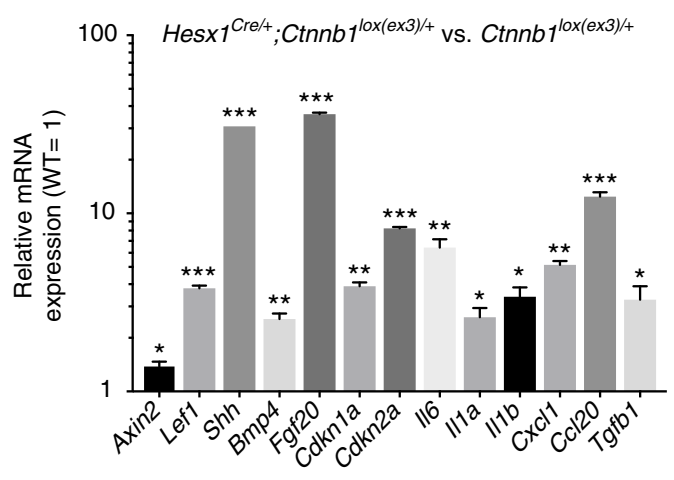

d

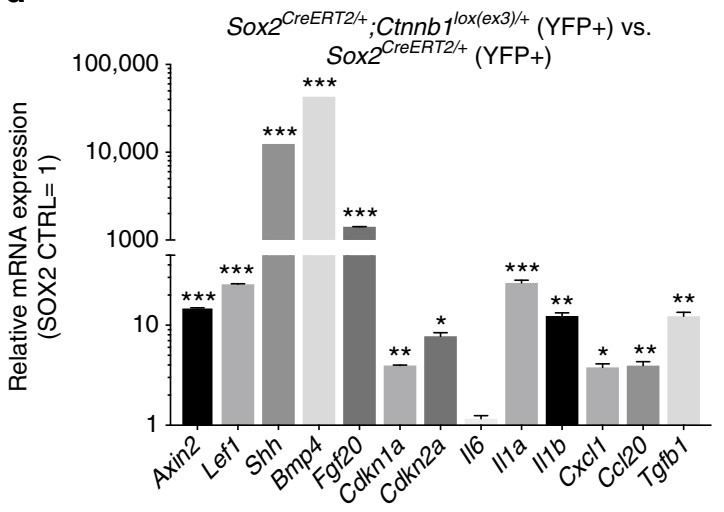

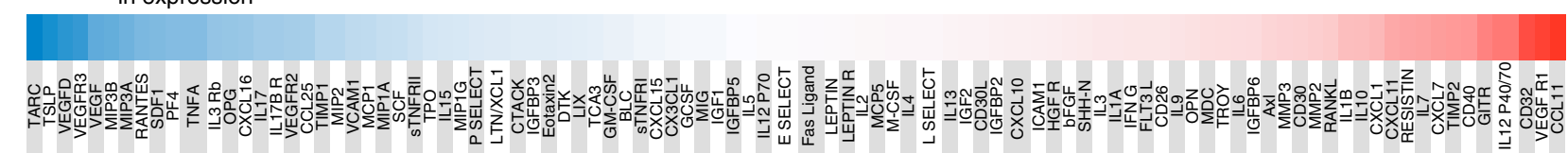

f

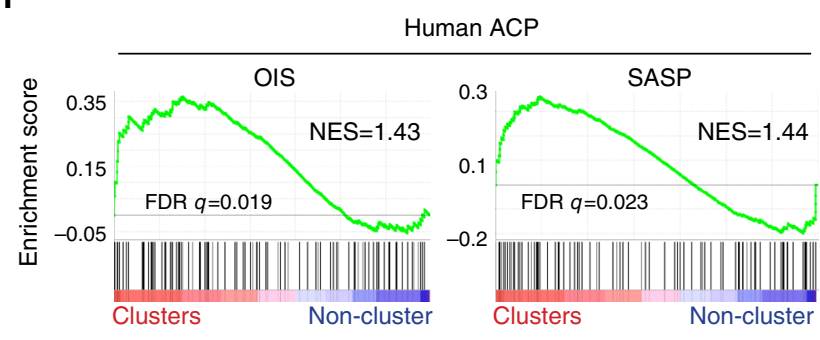

h

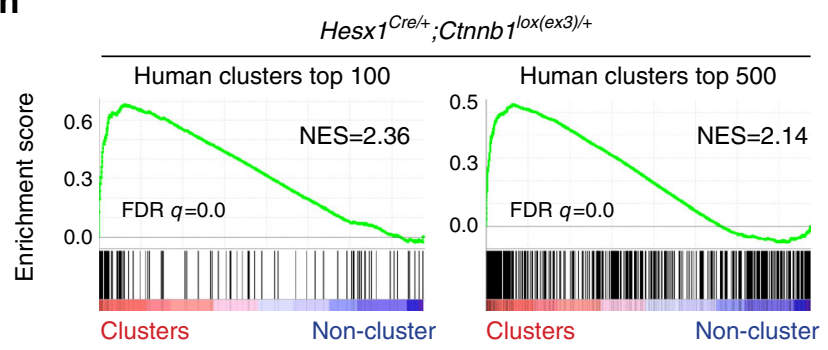

g

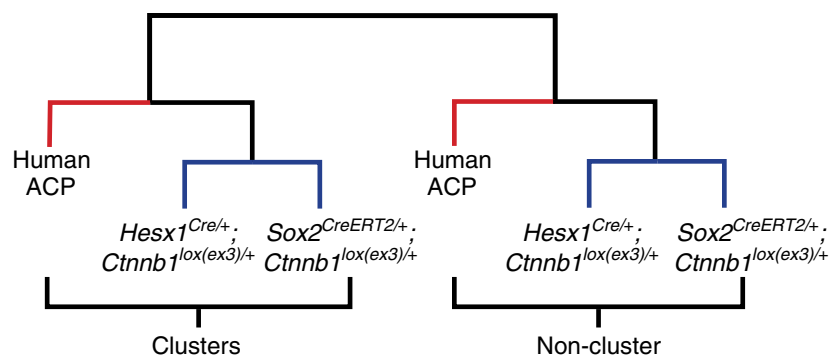

i

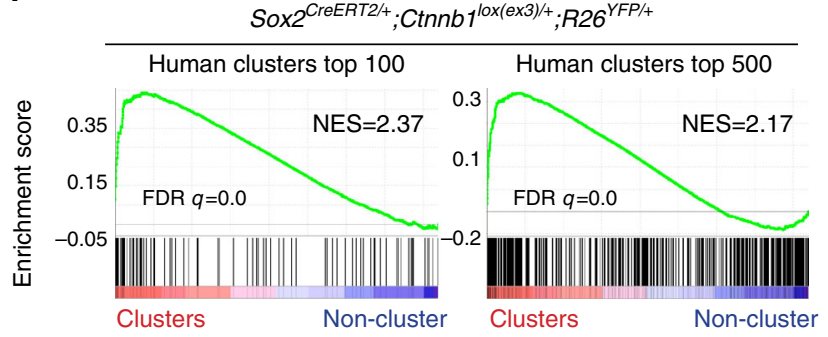




\section{Table 2 Reduced tumour-inducing potential in older vs.} young Sox2+ pituitary stem cells

$$
\text { Sox2 } 2^{\mathrm{CreERT2} /+^{*}} \text { C Ctnnb1 }{ }^{\mathrm{lox}(\mathrm{ex} 3) /+} ; \mathrm{R}^{\mathrm{YFP} /+}
$$

\begin{tabular}{lll}
\hline $\begin{array}{l}\text { Age at tamoxifen } \\
\text { induction }\end{array}$ & Tumour incidence $^{\mathbf{a}}$ & Number of mice \\
\hline 1 month & 15 (48 lesions) & 15 \\
$6-9$ months & 3 (5 lesions) & 17 \\
\hline *Unpaired $t$-test showed a statistically significant difference in tumour formation between age \\
$\begin{array}{l}\text { groups }(p=<0.0001) \\
\text { a Tumour incidence was assessed histologically at } 3 \text { months post-tamoxifen induction }\end{array}$ \\
\hline
\end{tabular}

to explore whether a genetic approach may impair the senescent/ SASP response more robustly. It has been shown that the strength of the senescence/SASP response depends on the oncogenic hit; e.g. the activation of the mitogen-activated protein kinase (MAPK) pathway by BRAF ${ }^{\mathrm{V} 600 \mathrm{E}}$ is a more potent senescence inducer than KRASG12V 51. We reasoned that the deletion of tumour suppressor Apc in $\operatorname{Hes}_{x 1}{ }^{\mathrm{Cre} /+} ; A p c^{f l / f l}$ mice would lead to the activation of the WNT pathway in embryonic precursors and the accumulation of wild-type $\beta$-catenin rather than its degradation-resistant form, which is encoded by the oncogenic Ctnnb1 locus in Hesx $1^{\mathrm{Cre} /+} ; \mathrm{Ctnn}_{\mathrm{b}} 1^{\mathrm{lox}(\mathrm{ex} 3) /+}$ mice. We expected wild-type $\beta$-catenin to be a weaker senescence inducer than oncogenic $\beta$-catenin, resulting in reduced senescence/SASP activation.

Hes $x 1^{C r e /+;} A p e^{f l / f l}$ mice were born at the expected Mendelian ratios, were normal and fertile with proper specification of pituitary endocrine lineages (Supplementary Data 4; Supplementary Fig. 8a). Although slight pituitary hyperplasia was consistently

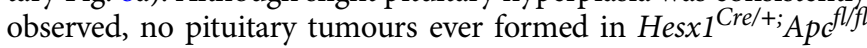
mice $(n=61)$, even when followed until 18 months of age $(n=17)$ (Fig. 7a; Supplementary Fig. 8a). Immunostaining studies revealed the presence of nucleo-cytoplasmic $\beta$-catenin-accumulating clusters in Hes $x 1^{\mathrm{Cre} /+;} A p \mathrm{c}^{f l / f l}$ embryos. These clusters resembled their oncogenic $\beta$-catenin counterparts in many ways, such as in the expression of SOX2, but not SOX9, and WNT pathway activation as evidenced by the over-expression of the WNT-target gene Axin2 (Fig. 7d; Supplementary Fig. 8b). Additionally, cluster cells in the $A p c$ mutant pituitary were negative for Ki67, phospho-Histone $\mathrm{H} 3$, or Caspase 3 and expressed the cell cycle inhibitors p21 and p16 (Fig. 7b; Supplementary Fig. 8c, d). Moreover, these clusters were strongly positive for DDR and NF- $\kappa \mathrm{B}$ activation markers such as phospho-DNA-PKcs, PARP-1 and phospho-I $\mathrm{K} \mathrm{B} \alpha$, indicative of the activation of a senescence programme (Fig. 7b; Supplementary Fig. 8d). Despite the similarities in gene expression, the average cluster size at 18.5 $\mathrm{dpc}$ in Hesx $1^{\mathrm{Cre} /+;} \mathrm{Ap} \mathrm{e}^{\text {flfl }}$ pituitaries was $1.97 \pm 1.36$ cells, rather than $5.89 \pm 0.33$ in Hesx $1^{\mathrm{Cre} /+} ; \mathrm{Ctnnb1} 1^{\text {lox }(e x \overline{3}) /+}$ embryos $\left(p<0.0001\right.$, Student's $t$ test; 126 clusters counted for Hes $x 1^{\mathrm{Cre} /+}$;

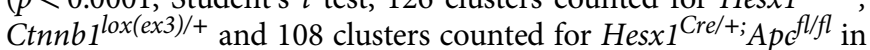
three different pituitaries).
qRT-PCR analysis comparing $H e s \times 1^{C r e /+;} A p c^{f l / f l}$ to $A p c^{f l /+}$ control pituitaries revealed no significant differences in the expression levels of several senescence and SASP factors including Cdkn1a, Cdkn2a, Shh, Bmp4, Illa, Illb, Il6, Cxcl1 and Tgfbl (Supplementary Fig. 8e). However when comparing expression levels of senescence/SASP factors relative to Hes $x 1^{\mathrm{Cre} /+;} \mathrm{Ctnn} b 1^{\text {lox }}$ $\left(\right.$ ex3)/ + pituitaries, these were reduced in $\mathrm{Hes} \times 1^{\mathrm{Cre} /+}$; $A p c^{f l f l}$ mutants (Fig. 7c), suggesting an overall reduction of the senescence/SASP response upon $A p c$ deletion and in line with the formation of smaller clusters. In situ hybridisation previously confirmed the upregulation of Axin2, Bmp4, Fgf3 and Shh in Hes $x 1^{C r e /+;} C t n n b 1^{l o x(e x 3) /+}$ clusters ${ }^{27}$. In contrast, despite Hes $x 1^{C r e /+}$;Ape ${ }^{f / f l}$ clusters expressing Axin2 and demonstrating WNT pathway activation, the expression of Bmp4, Fgf3 and Shh expression was not detectable (Fig. 7d). Moreover, the abundant SOX9+;EMCN+ cells surrounding the clusters in the Hes $\times 1^{\mathrm{Cre} /+}$; Ctnnb1 $1^{l o x(e x 3) /+}$ pituitaries (Fig. 4c) were not apparent in Hes $x 1^{\mathrm{Cre} /{ }_{+}} ; A p \mathrm{fl}^{\mathrm{fl} f \mathrm{l}}$ mutant glands (Fig. 7e), and overall quantification of the area covered by endomucin positive cells revealed values comparable to control littermates (Fig. 7f). In a Hes $\times 1^{\mathrm{Cre} /+}$; $A p c^{f l f l}$ pituitary where a larger cluster was identified, accumulation of EMCN+ cells was observed around it (Supplementary Fig. 8f), suggesting that a threshold of SASP factors may be needed to elicit the changes in tissue architecture. Finally, no tumours developed in Sox $2^{\mathrm{CreERT2/+}} ; A p c^{f l / f l}$ mice induced from 4 to 8 weeks of age in a follow up of up to a year ( $n=7$ mice). Together, these studies demonstrate that $A p c$ deletion in pituitary embryonic precursors/stem cells leads to reduced senescence/ SASP response and is not tumorigenic.

\section{Discussion}

In this paper, we show that the expression of oncogenic $\beta$-catenin and activation of the WNT pathway in Hesx1+ embryonic pituitary precursors or Sox $2+$ pituitary stem cells in young mice results in the activation of a robust SASP in targeted cells and the subsequent paracrine formation of tumours. These mouse tumours carry a low burden of somatic mutations that are mostly non-recurrent, which overlap with human ACP. To our knowledge, this is the first time that SASP activation is associated with non-cell autonomous cell transformation (i.e. induction of somatic mutations) and tumour initiation in an in vivo murine model of a human tumour. Of note, we refer to cell transformation to indicate the occurrence of somatic mutations not present in the germline and the acquisition of a proliferative advantage of the tumour relative to the non-tumour cells. However, mouse and human ACP do not harbour mutations in critical cancer genes (e.g. p53, p16, Myc, Braf, Egfr among others) suggesting that these tumours are not fully transformed. This is not surprising as human ACP is a benign tumour that very rarely progresses into a malignancy. A summary of the main findings of this study is shown in Fig. 8.

In the embryonic mouse ACP model, tumour progression does not start immediately after birth, but only after a latency period in

\footnotetext{
Fig. 5 Mouse and human $\beta$-catenin-accumulating clusters share a common signature of senescence and SASP. a-d Gene set enrichment analysis (GSEA)

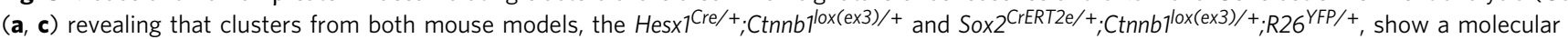
signature of oncogene-induced senescence (OIS) and senescence-associated secretory phenotype (SASP). qRT-PCR analysis showing the upregulation of several senescence and SASP factors in both mouse models $(\mathbf{b}, \mathbf{d})$. Bars in $\mathbf{b}$ and $\mathbf{d}$ represent the mean, and error bars represent standard error of the mean (SEM) of three biological replicates. ${ }^{\star} p<0.05,{ }^{* \star} p<0.01,{ }^{\star \star *} p<0.001$, Student's $t$ test. e Several cytokines are upregulated in the Hes $x 7^{C r e /+}$; Ctnnb $7^{\text {lox }(\mathrm{ex} 3) /+}$ mutant relative to the $\mathrm{Hesx}^{+/+} ; \mathrm{Ctnnb} 7^{\mathrm{lox}(\mathrm{ex} 3) /+}$ control pituitaries as measured in a mouse cytokine antibody array. $\mathbf{f}$ Gene set enrichment analysis (GSEA) demonstrating that laser-capture micro-dissected human clusters show a molecular signature of oncogene-induced senescence (OIS) and senescence-associated secretory phenotype (SASP). $\mathbf{g}$ Hierarchical clustering analysis of RNA-data sets from cluster and non-cluster cell populations indicating coherent grouping between specific cell compartments. h, i GSEA of the top 100 or 500 most upregulated genes in human clusters and the whole transcriptome of mouse clusters from both models demonstrating a common molecular signature
} 
a

Sox $2^{\text {CreERT2/+ }} ;$ Ctnnb $^{\text {lox }(e x 3) /+} ; R_{2} 6^{\text {YFP/+ }}$ and Sox $2^{\text {CreERT } 2 /+} ; R 26^{Y F P /+}$
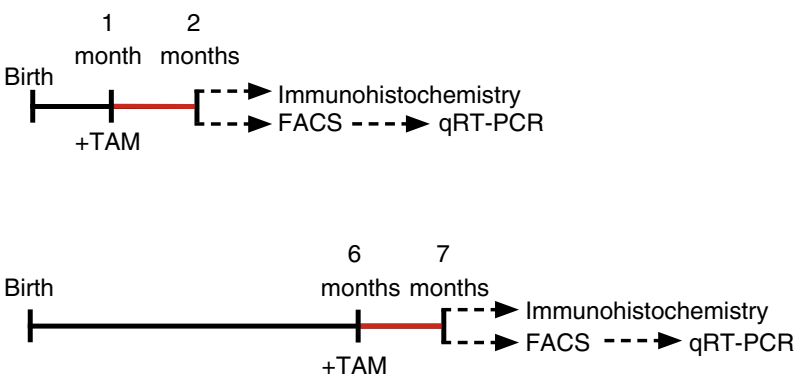

b
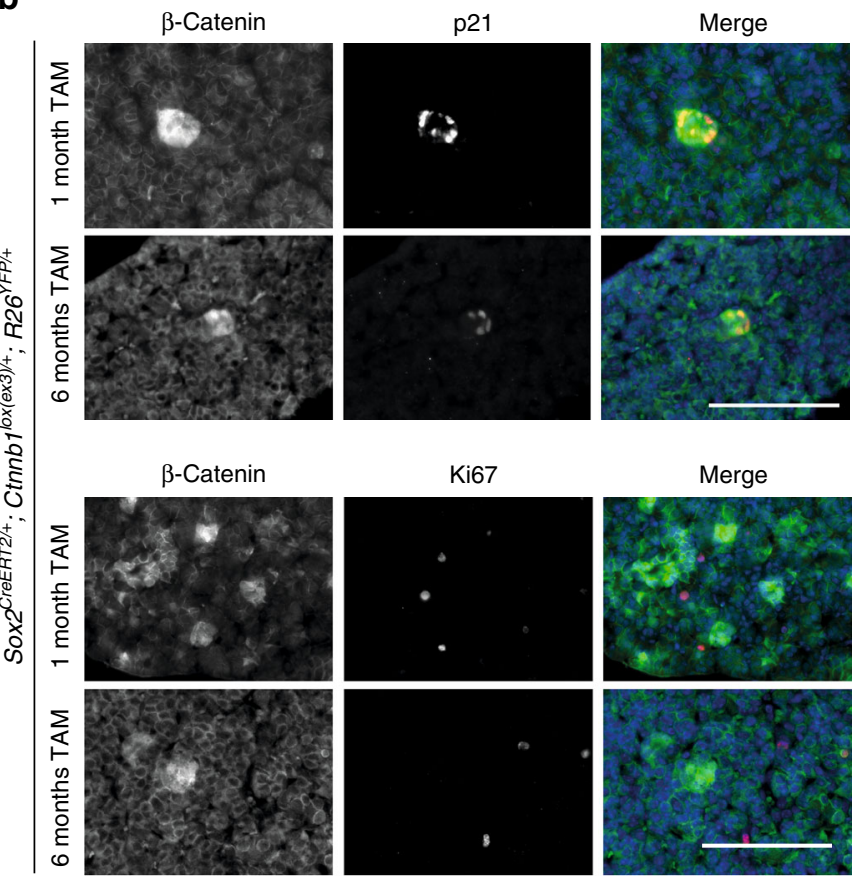

C

Sox $2^{\text {CreERT2/+ }} ; R_{2} 6^{\text {YFP+ }}$

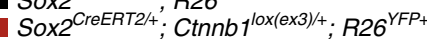

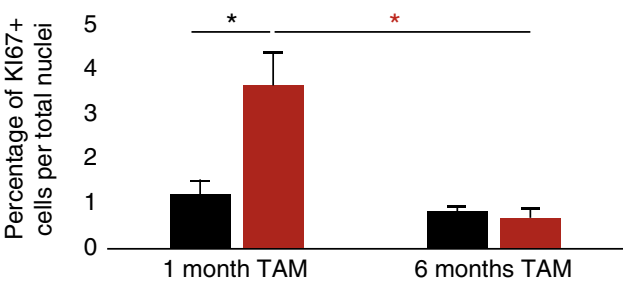

d

Sox2 $2^{\text {CreERT2/+ }} ;$ Ctnnb $^{\text {lox }(e \times 3) /+} ; R^{\text {R }} 6^{\text {YFP/+ }}$ vs. Sox2 ${ }^{\text {CreERT2/+ }} ; R^{\text {R }} 6^{\text {YFP/+ }}$ (6 months TAM)

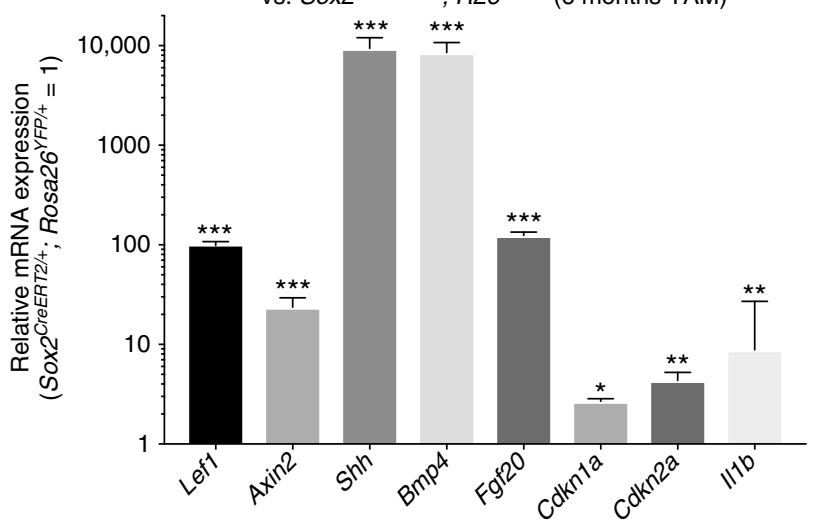

e

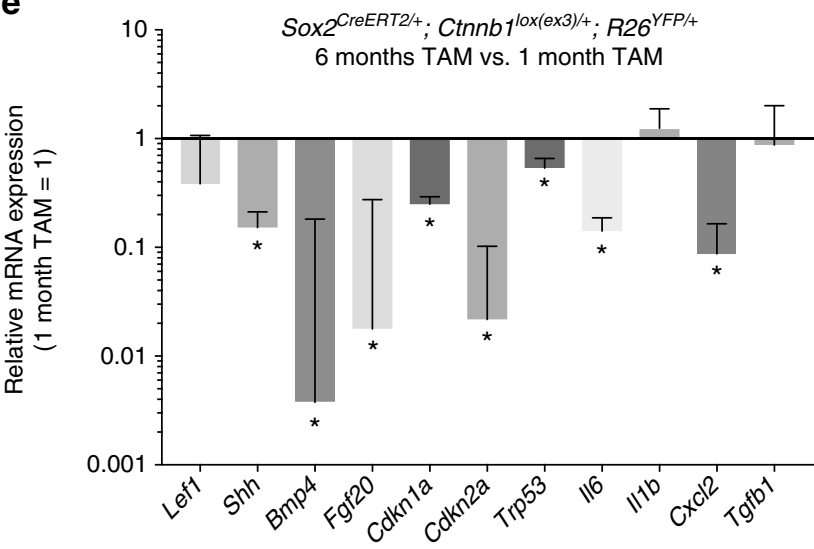

Fig. 6 Aged Sox $2+$ stem cells generate $\beta$-catenin-accumulating cell clusters with reduced senescence and SASP responses. a Schematic diagram of the

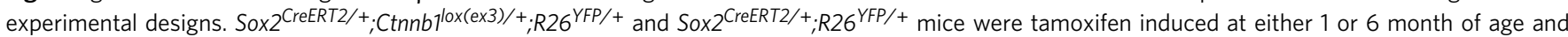
the anterior pituitary collected 4 weeks post induction and processed for histological and molecular analysis of YFP+ cells isolated by FACS ( $n=8$ mice per group). b Double immunostaining on histological sections of Sox $2^{\mathrm{CreERT2} /+} ;$ Ctnnb1 $1^{\text {lox(ex3)/+}} ;$ R26 $6^{\mathrm{YFP} /+}$ pituitaries revealing the presence of $\beta$-cateninaccumulating cell clusters positive for p21, but not for Ki67. Scale bar: $100 \mu \mathrm{m}$. c Quantitative analysis showing a significant higher Ki67 proliferation index

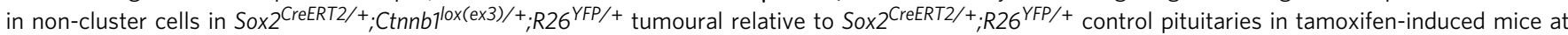

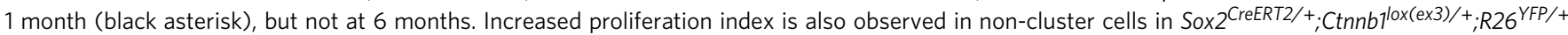
pituitaries of mice induced at 1 month relative to those induced at 6 months (red asterisk). d qRT-PCR analysis showing the upregulation of the WNT

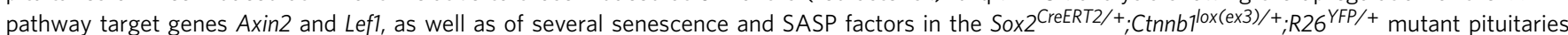
relative to the Sox2 $2^{\text {CreERT2/+; }}$ R26 $6^{\text {YFP/+ }}$ controls. e qRT analysis showing the relative expression of several senescence and SASP factors in Sox2 ${ }^{\text {CreERT2/+; }}$ Ctnnblox(ex3)/+$; R 26^{Y F P /+}$ mice tamoxifen induced at either 1 (young) or 6 (older) months of age. The expression of several senescent and SASP factors is significantly reduced in the older compared with the young mice. Bars in $\mathbf{c}, \mathbf{d}$ and $\mathbf{e}$ represent the mean, and error bars represent standard error of the mean (SEM) of three biological replicates. ${ }^{\star} p<0.05,{ }^{\star \star} p<0.01,{ }^{\star \star \star} p<0.001$, Student's $t$ test

which the pre-tumoural pituitary contains senescent clusters with an activated SASP. This non-linear growth dynamics have recently been characterised by magnetic resonance imaging ${ }^{37}$. The pre-tumoural pituitary also shows abnormal tissue architecture and overall slow cell proliferation. However, once rapidly dividing tumour cells appear, the clusters and most of the normal pituitary tissue are displaced. This growth pattern is compatible with the clusters' activities being required to initiate a process eventually leading to tumour initiation (hit-and-run model), rather than promoting tumour progression, which may be selfsustaining. Recently, it has been shown that senescence cells through SASP can induce dedifferentiation and stem cell marker expression $^{52}$. Transcriptomics failed to reveal the expression of Hesx1, but stem cell markers such as SOX9, Klf2, Klf4 and Cmyc 
a
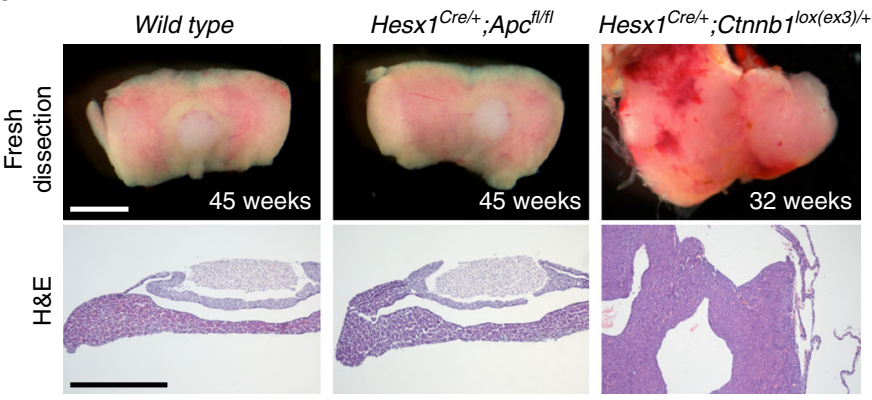

C

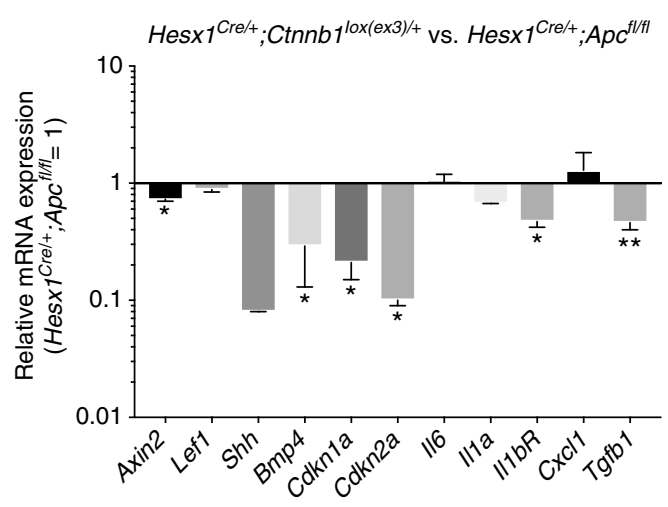

e

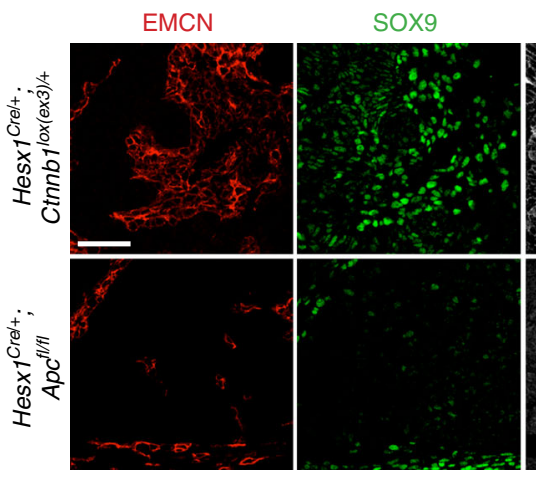

$\beta$-Catenin

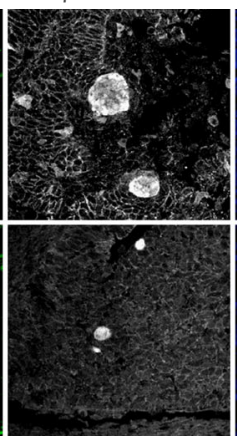

b

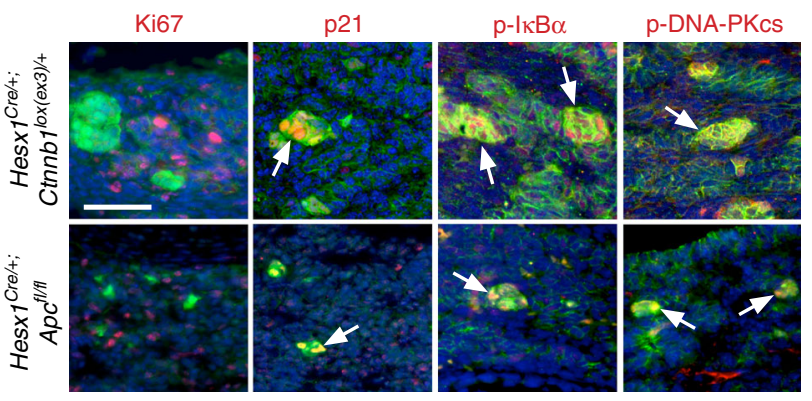

d

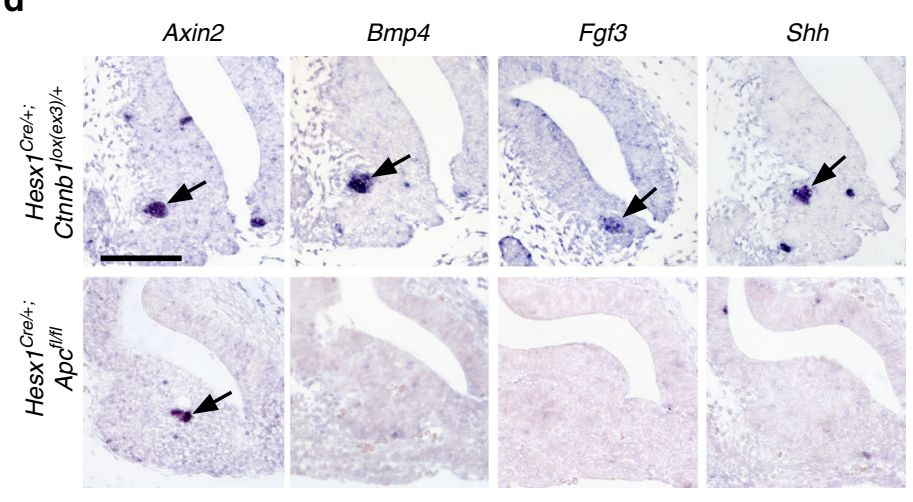

f

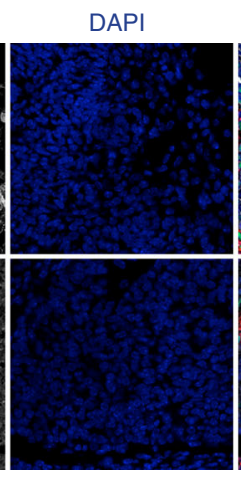

Merge

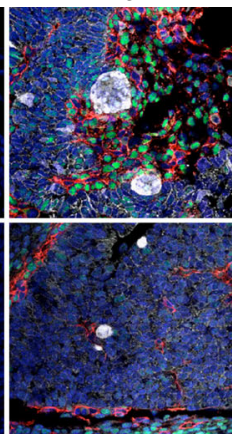

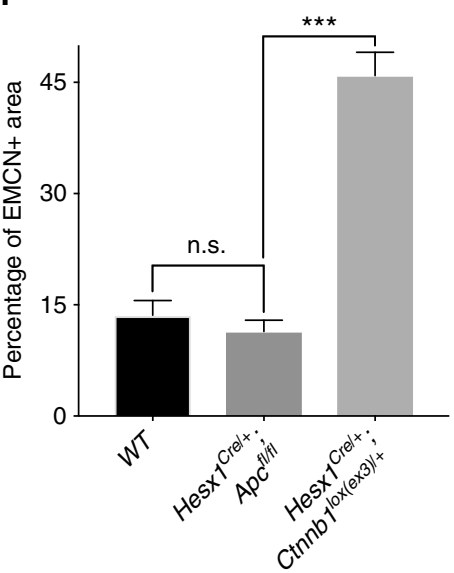

Fig. 7 Deletion of the tumour suppressor Apc results in $\beta$-catenin-accumulating senescent clusters with reduced SASP. a Brightfield and

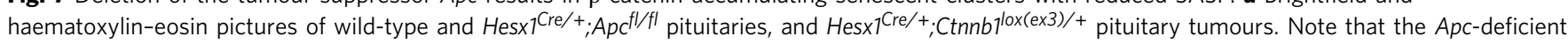
pituitary is enlarged but no tumours are apparent and it is histologically normal. The age of the mice is indicated in weeks. Scale bar: $1 \mathrm{~mm}$. $\mathbf{b}$ Double immunostaining revealing the expression of $\mathrm{p} 21$, phospho- $\mathrm{I} \mathrm{K} \alpha$ and phospho-DNA-PKcs in the $\beta$-catenin-accumulating clusters, indicating cell cycle

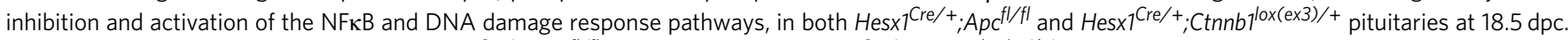

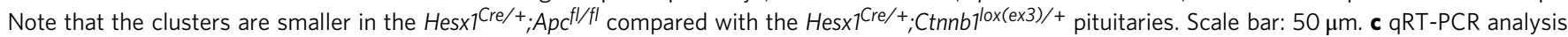

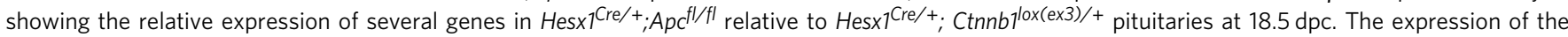
WNT pathway targets Axin2 and Lef1 are similar between the two genotypes, but several senescence and SASP factors show reduced expression levels in

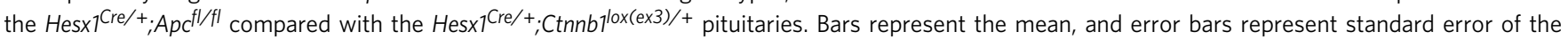
mean (SEM) of three biological replicates. ${ }^{\star} p<0.05,{ }^{* \star} p<0.01$, Student's $t$ test. $\mathbf{d}$ In situ hybridisation on histological sections of embryos at $14.5 \mathrm{dpc}$ showing the expression of Axin2 in small cell clusters and very reduced or absent detection of Bmp4, Fgf3 and Shh in the Apc-deficient pituitaries. Scale bar: $100 \mu \mathrm{m}$. e Triple immunostaining revealing the expression of $\beta$-catenin, SOX9 and EMCN. Note that the large areas of EMCN+;SOX9+ cells surrounding the

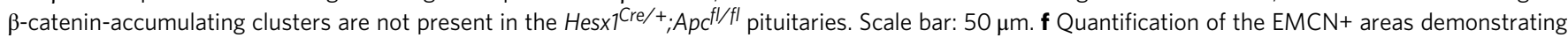
that wild-type and Apc-deficient pituitaries show similar levels, which are significantly increased in the Hesx $7^{\mathrm{Cre} /+} ; \mathrm{Ctnnb7}{ }^{\text {lox }(e \times 3) /+}$ pituitaries. Bars represent the mean, and error bars represent standard error of the mean (SEM) of three biological replicates. ${ }^{\star \star \star} p<0.001$, one-way ANOVA. All results are representative of at least three biological replicates 
a

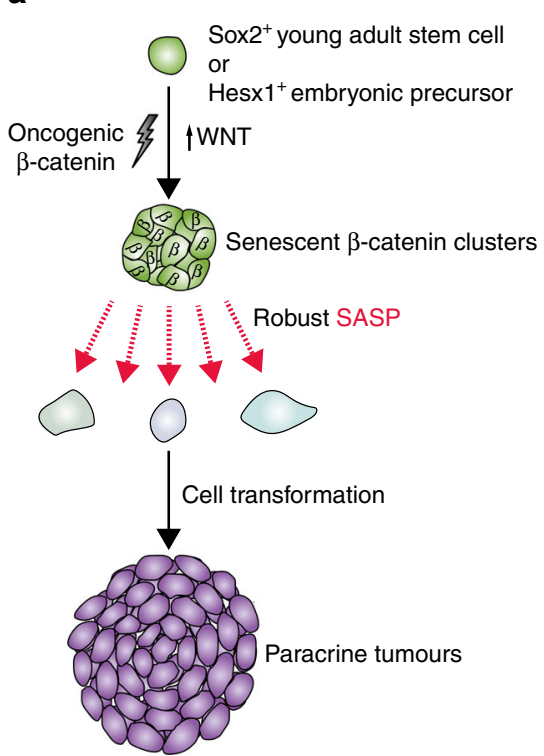

b

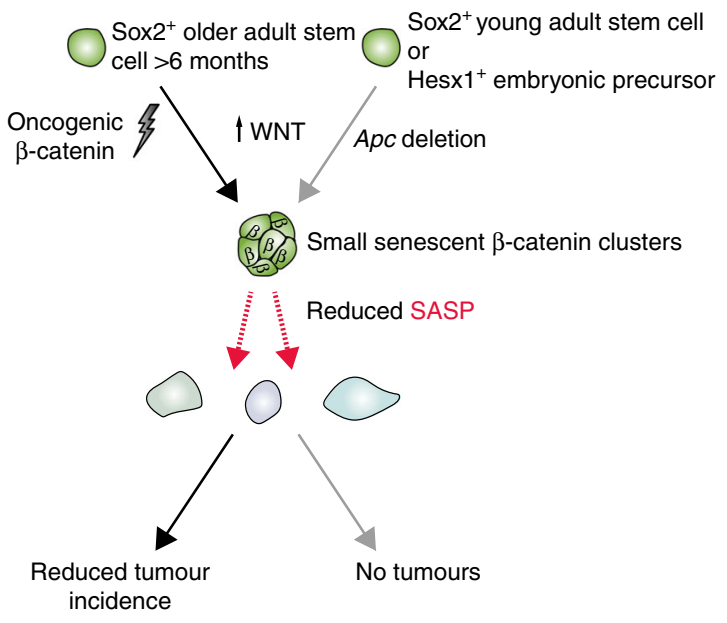

Fig. 8 Schematic summary of the main findings of this research. a Targeting Sox2+ stem cells from young adults, or Hesx1+ embryonic precursors to express oncogenic $\beta$-catenin, results in formation of senescent cell clusters and a robust SASP. This leads to cell transformation and generation of the cellof-origin of the paracrine tumours, thus the tumours are not derived from the mutation-sustaining cells. $\mathbf{b}$ In contrast, the expression of oncogenic $\beta$ catenin in Sox2+ stem cells of older mice leads to a reduced tumour incidence or the failure to produce tumours. In addition, upregulation of the WNT pathway through the deletion of Apc in young adult stem cells or embryonic precursors also fails to induce tumours. In all cases described in $\mathbf{b}$, there is attenuated senescence and SASP activation

were upregulated in mouse tumours (J.R. Apps and J.P. MartinezBarbera, manuscript submitted), sometimes in close proximity to the senescent cluster cells (i.e. SOX9; Fig. 4c) ${ }^{27}$. Immunostaining against SOX2 showed lack of expression of this stem cell marker in the murine tumours (Supplementary Fig. 9). In human ACP, the expression of stem cell markers (e.g. SOX2, SOX9, KLF4 and OCT4) has been found to be upregulated ${ }^{53}$. These results are compatible with a potential role of the senescent cluster cells in the promotion of reprogramming in vivo, a hypothesis that merits further research.

Elegant genetic experiments have shown that senescence is important to prevent tumourigenesis in mouse models of pituitary adenoma ${ }^{54}$. In agreement with this notion, our data suggest that senescence has an early cell-autonomous function that prevents the expansion of cluster cells harbouring oncogenic $\beta$ catenin. However, we reveal an additional non-cell autonomous role of senescent cells, mediated by the persistent activation of SASP resulting in paracrine cell transformation and tumourigenesis. Ablation of the senescent cluster cells at specific time points using chemical or genetic approaches will further help dissect their role in tumourigenesis. In particular, it will help determine when and for how long their activities are required for tumour initiation and/or maintenance.

We also reveal molecular similarities between the mouse and human clusters, which provide insights into the pathogenesis of human ACP. In contrast to the mouse tumours, the $\beta$-cateninaccumulating clusters are present in fully developed human ACP and tend to be located at the tips of finger-like epithelial protrusions that invade the brain ${ }^{36,37}$. Analysis of a human ACP xenograft model suggest that the activities of the clusters are important in orchestrating cell migration and tumour invasion ${ }^{36}$. We show that $\beta$-catenin clusters undergo senescence and initiate SASP during embryonic pituitary development in the mouse, and this supports the notion that childhood-onset ACP is a developmental tumour. This proposition is substantiated by the scrutiny of clinical data, which has revealed that paediatric ACP patients suffer significant height deficits around 12 months of age, many years before ACP diagnosis, suggesting potential growth hormone deficiency ${ }^{55}$. Of note, somatotrophs, which express growth hormone, are clearly defective in the Hes $x 1^{\mathrm{Cre} /+ \text {; }}$ Ctnnb $1^{\text {lox }(e \times 3) /+}$ ACP model by the end of gestation ${ }^{27}$. Therefore, clusters may develop embryonically in human patients and be present in the pituitary gland of the newborn prior to the appearance of any tumour mass. In fact, foetal ACPs displaying nucleo-cytoplasmic $\beta$-catenin-accumulating cells have recently been diagnosed in human foetus at 18 weeks ${ }^{56}$. Additionally, we show that the SASP response is attenuated with age in Sox2+ pituitary stem cells in older mice, which is in agreement with ACP being primarily a paediatric tumour ${ }^{29}$.

Paracrine tumourigenesis has been reported in other tumours and cancers, but so far not associated with senescence and SASP. Over-expression of platelet-derived growth factor (PDGF) (also a SASP factor) in neural precursors in mice and rats is sufficient to recruit normal neural stem and progenitor cells that acquired genetic aberrations and become malignant, capable of generating tumours upon transplantation ${ }^{57-59}$. Likewise, conditional deletion of Notch 1 in mouse skin epidermis ${ }^{60}$ or activation of $\beta$ catenin in hair follicles stem cells by deleting Ctnnb1 exon $3^{61}$ leads to over-activation of the WNT/ $\beta$-catenin pathway and induction of tumours/growths in a non-cell autonomous manner. Additionally, the expression of a constitutively active form of MEK1, activating the ERK MAPK pathway, in mouse epidermis results in skin polyps, which are mostly formed by cells not carrying oncogenic MEK1 ${ }^{62}$. More strikingly, in a mouse model for leukaemia the expression oncogenic $\beta$-catenin (i.e. Ctnnb1 deletion of exon3) in osteoblast precursors is sufficient to generate oncogenic hematopoietic stem cells capable of giving rise and propagating acute myeloid leukaemia ${ }^{63}$. Whether in these models the tumour-inducing cells are senescent and have activated SASP is an important aspect that merits further research. Our results support the possibility that the activities of the senescent cells result in changes in the cellular microenvironment driving genetic 
and possibly, epigenetic changes in neighbouring cells, which may fuel tumour initiation in some of these neoplasias.

In summary, this study provides evidence for a mechanism by which embryonic progenitors and postnatal stem cells can contribute to tumourigenesis in a non-cell autonomous manner, identifying senescence and SASP as mediators of this tumourinducing potential. Recent research has demonstrated that clearance of senescent cells, either naturally occurring in aged mice or diseased related, extends lifespan, improves the physiological function of several organs and delays tumourigenesis ${ }^{49}, 50,64$. Although the ablation of the cluster cells in the ACP mouse model was only partial, these results reinforce the idea that cluster cells are senescent and suggest that senolytic compounds may be of potential therapeutic interest against human ACP. The development of more specific senolytics capable of targeting a variety of senescent cells, including pituitary epithelial cells targeted with oncogenic $\beta$-catenin, will be required to further study the potential anti-tumourigenic effects of senescence ablation in the context of $\mathrm{ACP}$ and other tumours. In addition, further research is required to identify the pro- and anti-tumourigenic mediators in the SASP, and the mouse models presented here are suitable tools to dissect these opposite functions.

\section{Methods}

Mice and human samples of ACP. The Ctnnb1 $1^{\operatorname{lox}(e \times 3) /+}$, Sox $2^{\mathrm{CreERT2/+}}, \mathrm{Hes} \times 1^{\mathrm{Cre} /+}$, ROSA26 flox-stop-YFP/+,$A p c^{\text {flox/+ }}$ and $R O S A^{m T m G /+}$ mice have been previously described $28,48,65,66$. Sox $2^{\text {CreERT2/+}}$; Ctnnb1 $1^{\text {lox }(e x 3) /+} ;$ ROSA26 flox-stop-YFP/+ and So $2^{\text {CreERT2/+}}$; ROSA26 flox-stop-YFP/+ mice were induced with a single intra-peritoneal injection of tamoxifen (Sigma, T5648) at a dose of $0.15 \mathrm{mg}$ per gram of body weight. All mouse procedures were performed under licence, following UK Home Office Animals (Scientific Procedures) Act 1986 and local institutional guidelines (UCL ethical review committee). A humane end-point was chosen to avoid severe adverse effects and minimise suffering of mice harbouring tumours, according to UK Home Office and local Ethical Committee regulations. Samples of human ACP were obtained from the Department of Histopathology at Great Ormond Street Hospital for Children and the CCLG tissue bank with full consent (Research Ethics Committee application, REC 14 LO 2265).

Flow-activated cell sorting. Anterior pituitaries from age- and sex-matched mice were dissociated mechanically into single-cell suspensions following incubation in enzyme mix $(0.5 \% \mathrm{w} / \mathrm{v}$ Collagenase type 2 (Lorne Laboratories Ltd.), $0.1 \times$ Trypsin (Gibco) and $50 \mu \mathrm{g} / \mathrm{ml}$ DNaseI (Worthington) with $2.5 \mu \mathrm{g} / \mathrm{ml}$ Fungizone (Gibco) in Hank's Balanced Salt Solution (HBSS (Gibco) for $4 \mathrm{~h}$ at $37^{\circ} \mathrm{C}$. Cells were then washed in HBSS and suspended in PBS supplemented with 1\% Foetal Calf Serum (PAA), $25 \mathrm{mM}$ HEPES and $1 \mathrm{ng} / \mu \mathrm{l}$ of propidium iodide (PI, BD Pharmigen). Cells were flow sorted for YFP and PI fluorescence using a MoFlo XPD (Beckman Coulter, Fullerton, CA, USA) Flow Cytometer. Fluorescence was detected using a 530/540 filter, where PI positive cells were excluded and the YFP positive and negative fractions collected directly into RLT buffer (Qiagen) supplemented with $1 \%$ 2-mercaptoethanol (Sigma) for subsequent RNA isolation.

\section{Expression analyses in histological sections. Whole-mount X-gal staining,} immunostaining and in situ hybridisation on $4-6 \mu \mathrm{m}$ paraffin sections were performed as previously described ${ }^{27}$. At least three pituitaries or tumours and between two to six non-consecutive slides were analysed. As negative controls, sections were hybridised with sense riboprobes for in situ hybridisation or secondary antibody alone for immunohistochemistry/immunofluorescence. A list of the antibodies and the antigen retrieval methods used is presented in Supplementary Data 1.

EdU labelling. Pregnant females were injected intra-peritoneally with $100 \mathrm{mg} / \mathrm{kg}$ of EdU and embryos dissected 90 min later. After histological processing and sectioning (see above), double immunostaining for $\beta$-catenin and EdU was conducted using the Click-It EdU imaging kit (Invitrogen) according to the manufacturer's instructions.

RNA extraction and CDNA generation. Flow sorted cells from Sox $2^{\text {CreERT2/+; }}$ Ctnnb $1^{l o x(e x 3) /+} ; R O S A 26^{Y F P /+}$ and Sox $2^{\text {CreERT2/+}} ; R_{R O S A 26^{Y F P /+}}$ mice were processed for total RNA extraction using the RNeasy Micro kit (Qiagen). In total, $5 \mu \mathrm{l}$ of eluted RNA was then amplified using the Ovation Pico WTA V2 kit (NuGEN), followed by purification of the resultant cDNA using the QIAquick PCR purification kit (Qiagen). Pituitaries from Hes $x 1^{\text {Cre } /+} ;$ Ctnnb $1^{\text {lox }(e x 3) /+}$ and control littermates were dissected at $18.5 \mathrm{dpc}$. The posterior lobe of the pituitary was discarded and the intermediate and anterior lobes were processed for total RNA extraction using the RNeasy Micro kit (Qiagen). Approximately $1 \mu \mathrm{g}$ of total RNA was reverse transcribed to cDNA using the Transcriptor First Strand cDNA Synthesis Kit and random hexamers (Roche).

Quantitative real-time PCR and RNA-seq. Quantitative real-time PCR reactions were run in triplicate using the iTaq SYBR Green (BIORAD) and replicated using a minimum of four independent samples for each genotype. Primer sequences are included in Supplementary Data 5. For Shh, we used the Qiagen QuantiTect Primer Assay (Cat. no. 249900). Results were analysed using the $\Delta \Delta$ Ct method. For RNA-Seq of Sox2 $2^{\text {CreERT2/+}}$;Ctnnb1 $1^{\text {lox }(\text { ex } 3) /+} ;$ ROSA2 $6^{\text {YFP/+ }}$ and Sox $2^{\text {CreERT2/+; }}$ ROSA $26^{Y F P /+}$ flow-sorted YFP+ cells, isolated RNA was processed by the SMARTer V4 low input assay kit (Clontech) to generate amplified cDNA using the strand-switching protocol. Library preparation was performed with $200 \mathrm{pg}$ of amplified cDNA using the Nextera XT preparation kit with 12 cycles of PCR. Sequencing was then performed on an Illumina NextSeq 500 with 43 bp paired end reads. Sequencing data was then aligned in BaseSpace. FASTQ and BAM files are available on Array Express (E-MTAB-5538), Differential expression was performed using DESeq $2^{67}$. For GSEA, genes were ranked by the Wald statistic and GSEA performed using the pre-ranked tool in GSEA v2.2 (Broad Institute) ${ }^{68}$. The WNT pathway gene set was downloaded from the hallmark molecular signatures database v5.2 (Broad Institute) ${ }^{68}$.

Analysis of the relationship between murine and human ACP. To explore the relationship between the ACP models and human ACP, gene sets of the 100 and 500 most upregulated genes in human ACP were generated and GSEA performed as above. In addition, expression data (normalised counts or intensity values) of 11,347 genes, for which expression data was available between non-cluster and cluster cells from human ACP (array express accession no: E-MTAB-5266), So $2^{\text {CreERT2/+ }}$ Ctnnb1 ${ }^{\text {lox(ex3)}} ; R 26^{Y F P /+}$ tamoxifen-induced mice (E-MTAB-5538) and Hes $x 1^{\mathrm{Cre} /+} ;$ Ctnnbl $^{\text {lox(ex3)/+ }}(\mathrm{E}-\mathrm{MEXP} 3492)$ animals were median scaled and merged. Clustering was performed using Spearman's correlation and Ward's algorithm.

Exome sequencing of mouse tumours. Fully developed pituitary tumours and matched tail samples of 15 Hes $x 1^{\text {Cre } /+} ;$ Ctnnb 1 lox(ex3)/+ mice were collected at humane end-points. DNA was extracted using the DNeasy bloods and tissue kit (Qiagen) and exonic DNA was captured using the Agilent whole exome capture kit (SureSelect Mouse All Exon). Captured material was indexed and sequenced on the Illumina platform at the Wellcome Trust Sanger Institute at $30 \times$ depth. Raw pair end sequencing reads were aligned with BWA-mem to the GRCm38 mouse reference genome. Duplicated reads were marked using biobambam. Somatic variants were detected using CaVEMan, an expectation maximisation-based somatic substitution-detection algorithm. Detected somatic variants were then filtered using an array of quality filters and common mouse genome variants were excluded ${ }^{69}, 70$. A sub-selection of 14 genes, where mutant variant allele frequencies were greater than $20 \%$ and at least three mutant reads were detected, were validated by Sanger sequencing. Primer were designed in flanking regions (Supplementary Data 2). Sequencing of the PCR fragments was performed by Source BioScience (UK).

Semi-quantitative ELISA cytokine arrays. Mouse C1000 cytokine arrays (AAMCYT-1000-4, RayBiotech) were used to quantify the expression of SASP factors in protein extracts from whole pituitaries dissected at $18.5 \mathrm{dpc}$ according to manufacturer's instructions (sex was not determined in the embryos). For each experimental sample, total protein was extracted from four pituitaries using the cell lysis solution provided in combination with a TissueRuptor (Qiagen) and passed through a Qiashredder column (Qiagen). Total protein was then measured using BradfordUltra reagent (Expedeon). ELISA membranes were blocked for $30 \mathrm{~min}$ using blocking buffer provided by the manufacturer and then incubated overnight at $40{ }^{\circ} \mathrm{C}$ with $70 \mu \mathrm{g}$ of total protein. Following extensive washes, membranes were incubated with biotinylated antibodies and developed according to manufacturer's indications. Chemiluminescence detection was conducted with a ChemiDoc gel documentation system (BioRad) and imaged using ImageLab Software (Version 5.2.1, BioRad). Analysis of high-resolution images from each membrane was conducted using Fiji/ImageJ and the Protein Microarray Analysis macro (IRB, Barcelona). Calculation of fold changes in expression was conducted according to manufacturer's instructions.

Assessment of clonogenic potential. Anterior pituitaries were dissected and enzyme treated as described for flow-activated cell sorting, above. This was followed by mechanically dissociation into a single-cell suspension and subsequent washing in Hank's Balanced Salt Solution. Cells were placed directly into Adherent Pituitary Stem Cell medium (DMEM-F12; Gibco) containing 5\% Foetal Calf Serum (PAA), $20 \mathrm{ng} / \mathrm{ml}$ bFGF (R\&D Systems) and $50 \mathrm{ng} / \mathrm{ml}$ cholera toxin (Sigma). Cells were plated into six-well plates at a density of 1000, 2000 and 4000 cells per well for drug or vehicle treated. After seven days in culture, adherent colonies were fixed, washed with PBS and stained using Meyer's Haematoxylin (Sigma) for $10 \mathrm{~min}$ and counted manually. 
Ex vivo culture of mouse pituitaries and ABT-737 treatment. For ex vivo pituitary culture, anterior pituitaries were isolated from $\mathrm{Hes} \times 1^{\mathrm{Cre} /+} ; \mathrm{Ctnnb1} 1^{\text {lox }(\text { ex } 3) /+}$ embryos at $18.5 \mathrm{dpc}$, placed on top of $0.2 \mu \mathrm{M}$ Whatman filters (SLS) in 24 well plates containing $500 \mu \mathrm{l}$ of media (DMEM-F12 (Gibco), 1\% Pen/Strep (Sigma) and $1 \%$ FBS (PAA) supplemented with either ABT-737 at $2.5 \mu \mathrm{M}$ (Adooq Bioscience), ABT-263 at $1 \mu \mathrm{M}$ (Caymen Chemical) or vehicle. Anterior pituitaries were cultured for 3-4 days, changing media every day and subsequently processed for histological analysis.

Statistical analyses. Results are presented as means \pm s.e.m. unless otherwise indicated. One-tailed Student's $t$ test was performed to compare the means of two groups. Significance was assumed at $P<0.05$. Sample size varied, but a minimum of three biological replicas were used for analysis. Statistical analyses were performed using GraphPad software. No samples were excluded from analyses. No formal blinding or randomisation was used.

\section{Code availability. Codes are available upon request}

Received: 26 May 2017 Accepted: 31 October 2017

Published online: 28 November 2017

\section{References}

1. Campisi, J. Aging cellular senescence, and cancer. Annu. Rev. Physiol. 75, 685-705 (2013).

2. Serrano, M., Lin, A. W., McCurrach, M. E., Beach, D. \& Lowe, S. W. Oncogenic ras provokes premature cell senescence associated with accumulation of p53 and p16INK4a. Cell 88, 593-602 (1997).

3. Alcorta, D. A. et al. Involvement of the cyclin-dependent kinase inhibitorp16 (INK4a) in replicative senescence of normal human fibroblasts. Proc. Natl Acad. Sci. USA 93, 13742-13747 (1996).

4. Dhomen, N. et al. Oncogenic Braf induces melanocyte senescence and melanoma in mice. Cancer Cell 15, 294-303 (2009).

5. Di Micco, R. et al. Oncogene-induced senescence is a DNA damage response triggered by DNA hyper-replication. Nature 444, 638-642 (2006).

6. Coppe, J. P., Desprez, P. Y., Krtolica, A. \& Campisi, J. The senescenceassociated secretory phenotype: the dark side of tumor suppression. Annu. Rev. Pathol. 5, 99-118 (2010).

7. Maciel-Baron, L. A. et al. Senescence associated secretory phenotype profile from primary lung mice fibroblasts depends on the senescence induction stimuli. Age (Dordr.) 38, 26 (2016).

8. Kipling, D. et al. A transcriptomic analysis of the EK1.Br strain of human fibroblastoid keratocytes: the effects of growth, quiescence and senescence. Exp. Eye Res. 88, 277-285 (2009).

9. Coppe, J. P. et al. Senescence-associated secretory phenotypes reveal cellnonautonomous functions of oncogenic RAS and the p53 tumor suppressor. PLoS Biol. 6, 2853-2868 (2008).

10. Rodier, F. et al. Persistent DNA damage signalling triggers senescenceassociated inflammatory cytokine secretion. Nat. Cell Biol. 11, 973-979 (2009).

11. Chien, Y. et al. Control of the senescence-associated secretory phenotype by NF-kappaB promotes senescence and enhances chemosensitivity. Genes Dev. 25, 2125-2136 (2011).

12. Kuilman, T. et al. Oncogene-induced senescence relayed by an interleukindependent inflammatory network. Cell 133, 1019-1031 (2008).

13. Acosta, J. C. et al. A complex secretory program orchestrated by the inflammasome controls paracrine senescence. Nat. Cell Biol. 15, 978-990 (2013).

14. Tamm, I., Kikuchi, T., Cardinale, I. \& Krueger, J. G. Cell-adhesion-disrupting action of interleukin 6 in human ductal breast carcinoma cells. Proc. Natl Acad. Sci. USA 91, 3329-3333 (1994).

15. Tammela, T. et al. A Wnt-producing niche drives proliferative potential and progression in lung adenocarcinoma. Nature https://doi.org/10.1038/ nature22334 (2017)

16. Kang, C. et al. The DNA damage response induces inflammation and senescence by inhibiting autophagy of GATA4. Science 349, aaa5612 (2015).

17. Liu, D. \& Hornsby, P. J. Senescent human fibroblasts increase the early growth of xenograft tumors via matrix metalloproteinase secretion. Cancer Res. 67, 3117-3126 (2007).

18. Sparmann, A. \& Bar-Sagi, D. Ras-induced interleukin-8 expression plays a critical role in tumor growth and angiogenesis. Cancer Cell 6, 447-458 (2004).

19. Kang, T. W. et al. Senescence surveillance of pre-malignant hepatocytes limits liver cancer development. Nature 479, 547-551 (2011).

20. Yoshimoto, S. et al. Obesity-induced gut microbial metabolite promotes liver cancer through senescence secretome. Nature 499, 97-101 (2013).
21. Bavik, C. et al. The gene expression program of prostate fibroblast senescence modulates neoplastic epithelial cell proliferation through paracrine mechanisms. Cancer Res. 66, 794-802 (2006).

22. Krtolica, A., Parrinello, S., Lockett, S., Desprez, P. Y. \& Campisi, J. Senescent fibroblasts promote epithelial cell growth and tumorigenesis: a link between cancer and aging. Proc. Natl Acad. Sci. USA 98, 12072-12077 (2001).

23. Cahu, J., Bustany, S. \& Sola, B. Senescence-associated secretory phenotype favors the emergence of cancer stem-like cells. Cell Death Dis. 3, e446 (2012).

24. Canino, C. et al. SASP mediates chemoresistance and tumor-initiating-activity of mesothelioma cells. Oncogene 31, 3148-3163 (2012).

25. Ohanna, M. et al. Secretome from senescent melanoma engages the STAT3 pathway to favor reprogramming of naive melanoma towards a tumorinitiating cell phenotype. Oncotarget 4, 2212-2224 (2013).

26. Parrinello, S., Coppe, J. P., Krtolica, A. \& Campisi, J. Stromal-epithelial interactions in aging and cancer: senescent fibroblasts alter epithelial cell differentiation. J. Cell Sci. 118, 485-496 (2005).

27. Gaston-Massuet, C. et al. Increased Wingless (Wnt) signaling in pituitary progenitor/stem cells gives rise to pituitary tumors in mice and humans. Proc. Natl Acad. Sci. USA 108, 11482-11487 (2011).

28. Andoniadou, C. L. et al. Sox2(+) stem/progenitor cells in the adult mouse pituitary support organ homeostasis and have tumor-inducing potential. Cell Stem Cell 13, 433-445 (2013).

29. Muller, H. L., Merchant, T. E., Puget, S. \& Martinez-Barbera, J. P. New outlook on the diagnosis, treatment and follow-up of childhood-onset craniopharyngioma. Nat. Rev. Endocrinol. 13, 299-312 (2017).

30. Buslei, R. et al. Common mutations of beta-catenin in adamantinomatous craniopharyngiomas but not in other tumours originating from the sellar region. Acta Neuropathol. 109, 589-597 (2005).

31. Kato, K. et al. Possible linkage between specific histological structures and aberrant reactivation of the Wnt pathway in adamantinomatous craniopharyngioma. J. Pathol. 203, 814-821 (2004).

32. Sekine, S. et al. Craniopharyngiomas of adamantinomatous type harbor betacatenin gene mutations. Am. J. Pathol. 161, 1997-2001 (2002).

33. Brastianos, P. K. et al. Exome sequencing identifies BRAF mutations in papillary craniopharyngiomas. Nat. Genet. 46, 161-165 (2014).

34. Hofmann, B. M. et al. Nuclear beta-catenin accumulation as reliable marker for the differentiation between cystic craniopharyngiomas and rathke cleft cysts: a clinico-pathologic approach. Am. J. Surg. Pathol. 30, 1595-1603 (2006).

35. Holsken, A. et al. Adamantinomatous craniopharyngiomas express tumor stem cell markers in cells with activated Wnt signaling: further evidence for the existence of a tumor stem cell niche? Pituitary https://doi.org/10.1007/s11102 013-0543-8 (2013).

36. Stache, C. et al. Insights into the infiltrative behavior of adamantinomatous craniopharyngioma in a new xenotransplant mouse model. Brain Pathol. https://doi.org/10.1111/bpa.12148 (2014).

37. Boult, J. K. R. et al. Preclinical transgenic and patient-derived xenograft models recapitulate the radiological features of human adamantinomatous craniopharyngioma. Brain Pathol. https://doi.org/10.1111/bpa.12525 (2017).

38. Sharpless, N. E. \& Sherr, C. J. Forging a signature of in vivo senescence. Nat. Rev. Cancer 15, 397-408 (2015).

39. Zhu, J. \& You, C. Craniopharyngioma: survivin expression and ultrastructure Oncol. Lett. 9, 75-80 (2015).

40. Sharma, A., Singh, K. \& Almasan, A. Histone H2AX phosphorylation: a marker for DNA damage. Methods Mol. Biol. 920, 613-626 (2012).

41. Bensimon, A., Aebersold, R. \& Shiloh, Y. Beyond ATM: the protein kinase landscape of the DNA damage response. FEBS Lett. 585, 1625-1639 (2011).

42. $\mathrm{Li}, \mathrm{M}$. \& Yu, X. The role of poly(ADP-ribosyl)ation in DNA damage response and cancer chemotherapy. Oncogene 34, 3349-3356 (2015).

43. Debacq-Chainiaux, F., Erusalimsky, J. D., Campisi, J. \& Toussaint, O. Protocols to detect senescence-associated beta-galactosidase (SA-betagal) activity, a biomarker of senescent cells in culture and in vivo. Nat. Protoc. 4, 1798-1806 (2009).

44. Lee, B. Y. et al. Senescence-associated beta-galactosidase is lysosomal betagalactosidase. Aging Cell 5, 187-195 (2006).

45. Kurz, D. J., Decary, S., Hong, Y. \& Erusalimsky, J. D. Senescence-associated (beta)-galactosidase reflects an increase in lysosomal mass during replicative ageing of human endothelial cells. J. Cell Sci. 113, 3613-3622 (2000).

46. Traenckner, E. B. et al. Phosphorylation of human I kappa B-alpha on serines 32 and 36 controls I kappa B-alpha proteolysis and NF-kappa B activation in response to diverse stimuli. EMBO J. 14, 2876-2883 (1995).

47. Samulowitz, U. et al. Human endomucin: distribution pattern, expression on high endothelial venules, and decoration with the MECA-79 epitope. Am. J. Pathol. 160, 1669-1681 (2002).

48. Andoniadou, C. L. et al. Identification of novel pathways involved in the pathogenesis of human adamantinomatous craniopharyngioma. Acta Neuropathol. 124, 259-271 (2012). 
49. Yosef, R. et al. Directed elimination of senescent cells by inhibition of BCL-W and BCL-XL. Nat. Commun. 7, 11190 (2016).

50. Chang, J. et al. Clearance of senescent cells by ABT263 rejuvenates aged hematopoietic stem cells in mice. Nat. Med. 22, 78-83 (2016).

51. Kennedy, A. L. et al. Activation of the PIK3CA/AKT pathway suppresses senescence induced by an activated RAS oncogene to promote tumorigenesis. Mol. Cell 42, 36-49 (2011).

52. Mosteiro, L. et al. Tissue damage and senescence provide critical signals for cellular reprogramming in vivo. Science $354 \mathrm{https} / / /$ doi.org/10.1126/science. aaf4445 (2016).

53. Garcia-Lavandeira, M. et al. Craniopharyngiomas express embryonic stem cell markers (SOX2, OCT4, KLF4, and SOX9) as pituitary stem cells but do not coexpress RET/GFRA3 receptors. J. Clin. Endocrinol. Metab. https://doi.org/ 10.1210/jc.2011-2187 (2011).

54. Arzt, E., Chesnokova, V., Stalla, G. K. \& Melmed, S. Pituitary adenoma growth: a model for cellular senescence and cytokine action. Cell Cycle 8, 677-678 (2009).

55. Muller, H. L. et al. Longitudinal study on growth and body mass index before and after diagnosis of childhood craniopharyngioma. J. Clin. Endocrinol. Metab. 89, 3298-3305 (2004).

56. Kostadinov, S., Hanley, C. L., Lertsburapa, T., O'Brien, B. \& He, M. Fetal craniopharyngioma: management, postmortem diagnosis, and literature review of an intracranial tumor detected in utero. Pediatr. Dev. Pathol. 17, 409-412 (2014).

57. Uhrbom, L., Hesselager, G., Nister, M. \& Westermark, B. Induction of brain tumors in mice using a recombinant platelet-derived growth factor B-chain retrovirus. Cancer Res. 58, 5275-5279 (1998).

58. Fomchenko, E. I. et al. Recruited cells can become transformed and overtake PDGF-induced murine gliomas in vivo during tumor progression. PLoS ONE 6 , e20605 (2011).

59. Chen, Y. et al. Glioma initiating cells contribute to malignant transformation of host glial cells during tumor tissue remodeling via PDGF signaling. Cancer Lett. 365, 174-181 (2015).

60. Nicolas, M. et al. Notch1 functions as a tumor suppressor in mouse skin. Nat. Genet. 33, 416-421 (2003).

61. Deschene, E. R. et al. beta-Catenin activation regulates tissue growth non-cell autonomously in the hair stem cell niche. Science 343, 1353-1356 (2014).

62. Arwert, E. N. et al. Tumor formation initiated by nondividing epidermal cells via an inflammatory infiltrate. Proc. Natl Acad. Sci. USA 107, 19903-19908 (2010).

63. Kode, A. et al. Leukaemogenesis induced by an activating beta-catenin mutation in osteoblasts. Nature 506, 240-244 (2014).

64. Baker, D. J. et al. Naturally occurringp16(Ink4a)-positive cells shorten healthy lifespan. Nature 530, 184-189 (2016).

65. Shibata, H. et al. Rapid colorectal adenoma formation initiated by conditional targeting of the Apc gene. Science 278, 120-123 (1997).

66. Muzumdar, M. D., Tasic, B., Miyamichi, K., Li, L. \& Luo, L. A global doublefluorescent Cre reporter mouse. Genesis 45, 593-605 (2007).

67. Love, M. I., Huber, W. \& Anders, S. Moderated estimation of fold change and dispersion for RNA-seq data with DESeq2. Genome Biol. 15, 550 (2014).

68. Subramanian, A. et al. Gene set enrichment analysis: a knowledge-based approach for interpreting genome-wide expression profiles. Proc. Natl Acad. Sci. USA 102, 15545-15550 (2005).

69. Keane, T. M. et al. Mouse genomic variation and its effect on phenotypes and gene regulation. Nature 477, 289-294 (2011).

70. Jones, D. et al. cgpCaVEManWrapper: simple execution of CaVEMan in order to detect somatic single nucleotide variants in NGS data. Curr. Protoc. Bioinform. 56, 10.11-15.10.18 (2016).

\section{Acknowledgements}

We sincerely apologise for not including relevant publications due to editorial restrictions. We thank the Developmental Studies Hybridoma Bank (University of Iowa) and the National Hormone and Peptide Program (Harbor-University of California, Los Angeles Medical Center) for providing some of the antibodies used in this study. We thank Dr. Alicia Villa-Osaba for technical help with triple immunostaining experiments. This work was supported by the Medical Research Council (MRC) (Grants MR/ M000125/1 and MR/L016729/1), Great Ormond Street Hospital for Children Charity/ Children with Cancer UK (GOSHCC/CWCUK) (Grant W1055) and by the National Institute for Health Research Biomedical Research Centre at Great Ormond Street Hospital for Children National Health Service Foundation Trust and University College London. J.M.G.-M. is supported by a CONACYT-UCL Postgraduate Fellowship and a CONACYT-REDESD travel fellowship. S.H. and S.P. are supported by a Wellcome Trust Ph.D. Fellowship. J.R.A. is supported by a Cancer Research UK Clinical Research Training Fellowship. D.J.A. and M.R. are supported by Cancer Research UK, The Wellcome Trust and the ERC Synergy Programme. J.P.M.-B. is a Great Ormond Street Hospital for Children's Charity Principal Investigator.

\section{Author contributions}

J.M.G.-M. carried out the majority of the experiments, contributed to experimental design. S.H., G.C., S.P., S.N.M.-G. and C.S. performed experiments. J.A. and N.J. carried out the bioinformatics analysis on RNA sequencing data and contributed to the analysis of the exome sequencing data. G.K. carried out the immunostainings shown in Fig. 3. L. P. genotyped mice. A.G. performed the ablation experiments using the ABT senolytic compounds on IMR90 cells. A.V. and T.S.J. contributed to the pathological analysis of human samples. M.R. and D.J.A. performed the exome sequencing of mouse tumours and the bioinformatics analysis. C.L.A. performed and analysed experiments. J.G., C.R.G. and C.L.A. contributed to the overall experimental design and provided intellectual and strategic input. J.P.M.-B. designed experiments and overviewed the project. J.M.G.-M. and J.P.M.-B. wrote the manuscript.

\section{Additional information}

Supplementary Information accompanies this paper at https://doi.org/10.1038/s41467017-01992-5.

Competing interests: The authors declare no competing financial interests.

Reprints and permission information is available online at http://npg.nature.com/ reprintsandpermissions/

Publisher's note: Springer Nature remains neutral with regard to jurisdictional claims in published maps and institutional affiliations.

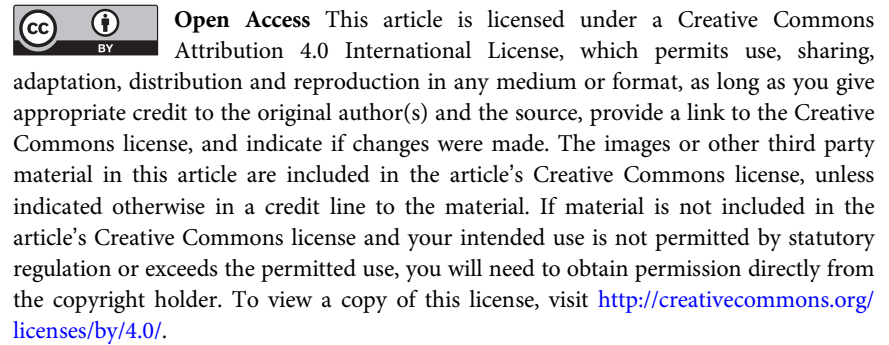
licenses/by/4.0/.

(c) The Author(s) 2017 